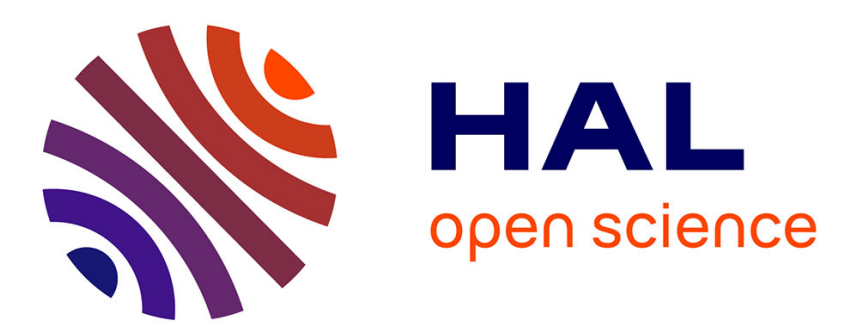

\title{
Design of Saturating State-Feedback with Sign-Indefinite Quadratic Forms
}

Isabelle Queinnec, Sophie Tarbouriech, Giorgio Valmorbida, Luca Zaccarian

\section{To cite this version:}

Isabelle Queinnec, Sophie Tarbouriech, Giorgio Valmorbida, Luca Zaccarian. Design of Saturating State-Feedback with Sign-Indefinite Quadratic Forms. IEEE Transactions on Automatic Control, 2022, 67 (7), pp.3507 - 3520. 10.1109/TAC.2021.3106878 . hal-03353776

\section{HAL Id: hal-03353776 \\ https://hal.laas.fr/hal-03353776}

Submitted on 24 Sep 2021

HAL is a multi-disciplinary open access archive for the deposit and dissemination of scientific research documents, whether they are published or not. The documents may come from teaching and research institutions in France or abroad, or from public or private research centers.
L'archive ouverte pluridisciplinaire HAL, est destinée au dépôt et à la diffusion de documents scientifiques de niveau recherche, publiés ou non, émanant des établissements d'enseignement et de recherche français ou étrangers, des laboratoires publics ou privés. 


\title{
Design of Saturating State-Feedback with Sign-Indefinite Quadratic Forms
}

\author{
I. Queinnec, S. Tarbouriech, G. Valmorbida, L. Zaccarian
}

\begin{abstract}
We propose a novel class of piecewise smooth Lyapunov functions leading to LMI-based stability/performance analysis and control design for linear systems with saturating inputs. We provide conditions for global properties, and also conditions for local properties and guaranteed estimates of the basin of attraction. The backbone of our result consists in using quadratic forms with constant matrices that are not necessarily sign definite, thereby providing additional degrees of freedom. Using generalized sector conditions involving the dead-zone nonlinearity and its derivative, we formulate convex optimization conditions to verify their positivity in the region of interest. Several numerical examples with connections to existing results, illustrate the potential behind our novel construction.
\end{abstract}

\section{INTRODUCTION}

The operating constraints of any practical control system often include magnitude limits on the actuators that must be taken into account in the control design to avoid potentially catastrophic effects (see, for example, [22] and [1]). The conservative (so-called low-gain) approach of restricting solutions to never reach the saturation bounds may limit excessively the system operation or induce poor performance. With linear plants, it is known that global asymptotic stability can be only achieved if the plant is not exponentially unstable (these plants are called Asymptotically Null Controllable with Bounded Controls - ANCBC) [21]. Moreover, some of these systems require nonlinear stabilizers [7]. When the plant is not ANCBC it is imperative to characterize the basin of attraction of the origin and to obtain tight estimates thus providing the user with a large set of operating conditions.

In the last two decades, constructive methods based on convex optimization led to direct design and anti-windup design strategies to mitigate performance degradation during saturation [11], [23], [31]. Most of these results use quadratic Lyapunov functions and the circle criterion to certify absolute stability. In this scenario, only the sector property of the saturation is considered and the resulting analysis is generally conservative. For the regional analysis and design which are necessary with non ANCBC plants, generalized sector conditions enjoyed by the saturation are used, typically leading to certified estimates of the basin of attraction based on sublevel sets of the Lyapunov functions. When using the Popov

I. Queinnec, S. Tarbouriech and L. Zaccarian are with LAAS CNRS, University of Toulouse, CNRS, Toulouse, France, Email: \{tarbour,queinnec,zaccarian\}@laas.fr. Luca Zaccarian is also with the Dept. of Industrial Engineering, University of Trento, Italy. G. Valmorbida is with Laboratoire des Signaux et Systèmes, CentraleSupélec, CNRS, Université Paris-Saclay and with Inria Saclay, 3 Rue Joliot-Curie, Gif-surYvette 91192, France, Email: giorgio.valmorbida@12s.centralesupelec.fr.

Research supported in part by ANR via grant HANDY, number ANR-18CE40-0010. criterion [14], the nonlinearity is incorporated in the Lyapunov function and less conservative regional results can be obtained using information on the gradient of the nonlinearity [4], [17]. However it is only recently that the sector properties of the nonlinearity have been used to also relax the positivity of the Lyapunov functions for optimization-based analysis of Lure systems [6], [16], [26], [27].

In this paper, we introduce a new class of non-quadratic Lyapunov functions for stability analysis and control design providing global and non-global guarantees. The quadratic forms that we consider were first introduced in [19] and then revisited in [4] and [16]. However, in [4], [19] a constraint on the positivity of the matrix in the quadratic form was imposed. As in the analysis results of [16], we exploit here the flexibility of matrices that are not sign definite for the stability analysis and, for the first time, for the control synthesis of nonlinear state feedback laws for saturating systems. Both the global and regional cases are studied. In the framework of Linear Complementarity Systems, a similar Lyapunov function was presented in [2]. Moreover, in the discrete-time saturated systems context, [10] used a special case of the function studied here. Not many results focus on non-positive quadratic forms when addressing continuous-time saturated systems. In our recent work [27], we show that relaxing the positivity conditions allows improving the nonlinear $\mathcal{L}_{2 m}$ gain estimates, when using piecewise polynomial storage functions. Also [16] suggested similar positivity relaxations when focusing on max of quadratics for analysis purposes. Additionally, for systems with slope bounds in a known set of the state space, regional gain and regions of attractions were studied in [26]. Finally, for systems with rational Jacobian one can circumvent the need for a pre-defined set in which the slope is bounded [6].

In this paper we propose synthesis conditions exploiting sign-indefinite quadratic forms. For the global case, our conditions are authentic linear matrix inequalities, and allow constructing certificates for examples where quadratic functions are proven to not exist. For the regional case we obtain suggestive functions whose positivity is only guaranteed in the estimated region of attraction, and which even become negative outside that certified domain, so that extra degrees of freedom are available for obtaining nonconservative (and nonconvex) contractive sublevel sets. An interesting parallel approach is provided in [15], where generalized quadratic forms are used to address the analysis problems considered in our paper. In addition to also providing design conditions (whereas only analysis is addressed in [15]), our conditions directly imply well posedness of the nonlinear algebraic loop, whereas this property is stated as an assumption in [15].

The paper is organized as follows. Section II describes 
the system under consideration and the analysis and design problems, together with some background material. Section III introduces our new sign-indefinite quadratic form and the arising Lyapunov functions, together with some new tools for establishing its positivity in the region of interest. The paper is completed by Section IV providing global analysis and design conditions, and Section $\mathrm{V}$ providing regional analysis and design conditions. Several examples taken from the literature are presented throughout the paper.

Notation. $\mathbb{R}^{n}$ is the $n$-dimensional Euclidean space, while $\mathbb{C}$ is the complex plane and $\Re(s)$ is the real part of number $s \in \mathbb{C}$. $\mathbb{S}_{\geq 0}^{n}$ (respectively $\mathbb{S}_{>0}^{n}$ ) is the sets of symmetric positive semi-definite (respectively, positive definite) matrices of dimension $n . \mathbb{D}_{>0}^{m}$ (respectively $\mathbb{D}_{>0}^{m}$ ) is the set of diagonal positive semi-definite (respectively, positive definite) matrices of size $m$. Given any symmetric matrix $A, \lambda_{m}(A)$ and $\lambda_{M}(A)$ are, respectively, its minimum and maximum real eigenvalues. For any matrix $A, A^{\top}$ denotes its transpose and $\operatorname{He}(A)=A+A^{\top}$. For any square matrix $A, \operatorname{Tr}(A)$ denotes its trace. For $q$ matrices $A_{i}, i=1, \ldots, q$, $\operatorname{diag}\left(A_{1}, \ldots, A_{q}\right)$ denotes the block-diagonal matrix constructed from matrices $A_{i} . I_{n}$ denotes the identity matrix of dimension $n$, whereas 0 stands for a matrix of zeros of appropriate dimensions. For any $y \in \mathbb{R}^{m},|y|_{\infty}$ and $|y|$ stand respectively for its infinity and Euclidean norms.

\section{Problem Statement}

\section{A. Linear state feedback with deadzone loop}

Consider the following plant

$$
\dot{x}=A x+B \operatorname{sat}(u)
$$

where $x \in \mathbb{R}^{n}$ and $u \in \mathbb{R}^{m}$ are the state and the input, respectively. $A$ and $B$ are constant matrices of appropriate dimensions and pair $(A, B)$ is supposed to be stabilizable. Function sat $: \mathbb{R}^{m} \rightarrow \mathbb{R}^{m}$ denotes the vector-valued symmetric decentralized unit saturation, whose components are defined for $i=1, \ldots, m$ as $\operatorname{sat}_{i}\left(u_{i}\right):=\max \left\{-1, \min \left\{1, u_{i}\right\}\right\}$, with $u_{i}$ being the $i$-th component of vector $u$. Model (1) well represents situations where the saturation limits are not unitary by simply rescaling the columns of $B$.

By defining the dead-zone function as $\operatorname{dz}(u):=u-\operatorname{sat}(u)$, system (1) reads:

$$
\dot{x}=A x+B u-B \mathrm{dz}(u) .
$$

In this paper we are interested in state-feedback control laws defined by

$$
u(x)=K_{1} x+K_{2} \mathrm{dz}(u(x)) .
$$

where $K_{1} \in \mathbb{R}^{m \times n}$ and $K_{2} \in \mathbb{R}^{m \times m}$ are either known in the problem of stability analysis or to be designed in the synthesis problem.

The presence of the dead-zone at the right-hand side of (3) induces a nonlinear algebraic loop implicitly defining a piecewise affine feedback selection $x \mapsto u(x)$. By setting $K_{2}=0$ one retrieves the classical linear state-feedback case without algebraic loop [23]. If the algebraic loop is wellposed then one can guarantee the existence of a (piecewise affine) solution to (3). Necessary and sufficient well-posedness conditions can be for example derived from [12, Claim 2].

The following facts are straightforward consequences of [30, Prop. 1] and [12, Claim 1 and Remark 1], respectively.

Fact 1: If there exists $\Delta \in \mathbb{D}_{>0}^{m}$ such that $2 \Delta-\Delta K_{2}-$ $K_{2}^{\top} \Delta>0$, then the algebraic loop in (3) is well-posed.

Fact 2: If the algebraic loop in (3) is well-posed, then its unique solution is a globally Lipschitz piecewise affine function of $x$ over a finite polytopic partition of $\mathbb{R}^{n}$.

\section{B. Problem statement and sector conditions background}

The closed-loop system resulting from the interconnection of (2) and (3) is given by

$$
\begin{aligned}
\dot{x} & =\left(A+B K_{1}\right) x+B\left(K_{2}-I_{m}\right) \mathrm{dz}(u(x)) \\
u(x) & =K_{1} x+K_{2} \mathrm{dz}(u(x)) .
\end{aligned}
$$

Due to the presence of the dead-zone, the closed-loop dynamics (4) is nonlinear and asymptotic stability of the origin can be ensured globally (that is for any initial condition $x(0) \in \mathbb{R}^{n}$ ) or only locally (that is, only for initial conditions in a neighborhood of the origin). Fundamental limitations of bounded stabilization of linear systems [20] imply that when $A$ has exponentially unstable eigenvalues, the maximal set of initial conditions providing solutions that converge to zero (the so-called basin of attraction) is bounded. The exact characterization of the basin of attraction of the origin remains an open problem in general. Hence, a challenging problem consists in computing accurate estimates. Whenever we can provide a guarantee on the size of the basin of attraction (typically through an inner approximation), we talk about regional asymptotic stability (as opposed to merely local asymptotic stability, where the basin of attraction may be arbitrarily small). Feedback (4) provides an interesting context where (due to the properties of function dz) local asymptotic stability holds if and only if $A+B K_{1}$ is Hurwitz, but the characterization of a large estimate (namely an inner approximation) of the basin of attraction requires nontrivial derivations (see, e.g., [11], [23], [31]).

In this paper we use piecewise quadratic Lyapunov functions stemming from sign-indefinite quadratic forms with the goal in mind of addressing both the global and the regional exponential stability problems in the two cases where $K_{1}$ and $K_{2}$ are fixed (analysis) or to be designed (synthesis).

In the global case, we address the following problems.

Problem 1 (Global Analysis): Given gains $K_{1}, K_{2}$ in (4), determine whether the origin is globally exponentially stable.

Problem 2 (Global Synthesis): Compute gains $K_{1}, K_{2}$ in (4) ensuring global exponential stability of the origin.

We address Problems 1 and 2 by exploiting the following well-known global sector condition (see, for example, [14]).

Fact 3 (Global Sector Condition): The inequality

$$
\mathrm{dz}(u)^{\top} T(u-\mathrm{dz}(u)) \geq 0
$$

holds for any $u \in \mathbb{R}^{m}$ and for any $T \in \mathbb{D}_{\geq 0}^{m}$.

In the regional case, we address the following problems.

Problem 3 (Regional Analysis): Given gains $K_{1}, K_{2}$ in (4), determine whether the origin is locally exponentially stable and provide an estimate of its basin of attraction. 


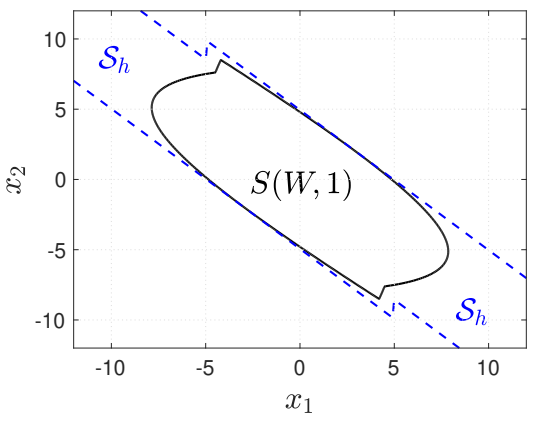

Fig. 1. Regional stability via inclusion of a sub-level set $S(V, 1)$ of function $V$ within set $\mathcal{S}_{h}$.

Problem 4 (Regional Synthesis): Compute gains $K_{1}, K_{2}$ in (4) ensuring local exponential stability of the origin, together with an estimate of the basin of attraction.

To study regional properties it is useful to generalize the global sector condition of Fact 3 in ways originally characterized in [9], [11]. As shown in Figure 1, regional asymptotic stability is assessed by focusing on an open sublevel set $S(W, 1):=\left\{x \in \mathbb{R}^{n}: W(x)<1\right\}$ of a Lyapunov function $W$ restricted to the set

$$
\mathcal{S}_{h}:=\left\{x \in \mathbb{R}^{n}:|h(x)|_{\infty} \leq 1\right\},
$$

where function $h: \mathbb{R}^{n} \rightarrow \mathbb{R}^{m}$ is typically vanishing at the origin. Most existing works [9], [11], [12], [23] use linear selections for $h$. In this paper we use the following implicit form, where $H_{1}$ and $H_{2}$ are arbitrary design parameters and function $x \mapsto u(x)$ is implicitly defined in (3) and characterized in Facts 1 and 2,

$$
h(x)=H_{1} x+H_{2} \mathrm{dz}(u(x)) .
$$

With the above selections, the following result is a direct adaptation of [24, Lemma 1] (see also [13]).

Fact 4 (Regional Sector Condition): The inequality

$$
\mathrm{dz}(u)^{\top} T(u-\mathrm{dz}(u)-h(x)) \geq 0
$$

holds for all $x \in \mathcal{S}_{h}$, all $u \in \mathbb{R}^{m}$ and all $T \in \mathbb{D}_{>0}^{m}$.

We complete our background material by introducing a few equalities inspired by the bounds initially proposed in [4], exploiting the properties of the directional derivative $x \mapsto \dot{u}(x)$ of function $x \mapsto u(x)$ in (3) and the directional derivative $x \mapsto \mathrm{dz}(u(x))$ of function $x \mapsto \mathrm{dz}(u(x))$ along the solutions of (4).

Fact 5 (Derivative of the dead-zone): The identities

$$
\begin{aligned}
& \dot{\mathrm{d} z}(u(x))^{\top} T(\dot{u}(x)-\mathrm{d} z(u(x))) \equiv 0, \\
& \mathrm{~d} z(u(x))^{\top} T(\dot{u}(x)-\dot{\mathrm{d} z}(u(x))) \equiv 0,
\end{aligned}
$$

hold for almost all $x \in \mathbb{R}^{n}$, and for all $T \in \mathbb{D}^{m}$.

Proof. We prove the relations for all $x$ such that $\left|u_{i}(x)\right| \neq 1$, for all $i=1, \ldots, m$. For those values of $x$ the proof follows by noting that each component of $u$ satisfies

$$
\dot{\mathrm{dz}}_{i}\left(u_{i}(x)\right)=\left\{\begin{array}{ccc}
\dot{u}_{i}(x) & \text { if } & \left|u_{i}(x)\right|>1 \\
0 & \text { if } & \left|u_{i}(x)\right|<1,
\end{array}\right.
$$

and

$$
\dot{u}_{i}(x)-\dot{\mathrm{d}} \mathrm{z}_{i}\left(u_{i}(x)\right)=\left\{\begin{array}{ccc}
0 & \text { if } & \left|u_{i}(x)\right|>1 \\
\dot{u}_{i}(x) & \text { if } & \left|u_{i}(x)\right|<1 .
\end{array}\right.
$$

Remark 1: The control solution (4), requires the knowledge of the physical saturation output sat $\mathrm{ph}_{\mathrm{ph}}$ present in the actuators, which might not be accessible in industrial applications. In this setting, a workaround is the introduction of an artificial (conservative) saturation function sat at the controller output, so that the actuation effort reaching the plant is $\operatorname{sat}_{\mathrm{ph}}(\operatorname{sat}(u))=\operatorname{sat}(u)$, where the last equality holds as long as the artificial saturation levels of sat are smaller than or equal to the physical saturation levels of $s t_{p h}$. In this case, the artificial saturation provides an effective way to obtain the closed loop (4) even when the output of $\mathrm{sat}_{\mathrm{ph}}$ is not accessible.

Besides this fact, mismatches between the saturation model sat and the actual saturation nonlinearity sat ${ }_{\mathrm{ph}}$ may also emerge. In this case we emphasize that the Lyapunov-based results presented in this paper enjoy intrinsic robustness of asymptotic stability (see, e.g., [8, Chapter 7]), so that a certain level of nonzero mismatch is guaranteed to preserve the asymptotic stability properties in a semiglobal practical sense (see [8, Def. 7.18]). Due to this robustness-in-thesmall guarantee, we expect some kind of graceful performance degradation when the saturation modeling is imprecise (e.g., the slope of the saturation may be not unitary in certain ranges) and in cases where the physical saturation levels might be smaller than the artificial ones (indeed in those cases $\left.\operatorname{sat}_{\mathrm{ph}}(\operatorname{sat}(u)) \neq \operatorname{sat}(u)\right)$. Similarly, due to the stated robustness-in-the-small, it is straightforward to extend our proofs to the case where the state measurement used in our feedback is replaced by an estimated state provided by a sufficiently fast Luenberger observer. Providing quantitative bounds on the tolerated mismatch that does not destroy stability, namely a robustness-in-the-large guarantee, is possible by embedding the nonlinearities in suitable inflated sector conditions, but is beyond the scope of this work and provides an interesting direction of future investigation.

\section{EXTENDED SIGN INDEFINITE LYAPUNOV FUNCTION}

\section{A. Proposed structure}

The four main results of this paper, providing solutions to Problems 1-4, rely on a generalization of the piecewise smooth Lyapunov function originally proposed in [4], corresponding to

$$
\begin{aligned}
V(x) & =\left[\begin{array}{c}
x \\
\mathrm{dz}(u(x))
\end{array}\right]^{\top}\left[\begin{array}{ll}
P_{11} & P_{12} \\
P_{12}^{\top} & P_{22}
\end{array}\right]\left[\begin{array}{c}
x \\
\mathrm{dz}(u(x))
\end{array}\right] \\
& =\left[\begin{array}{c}
x \\
\mathrm{dz}(u(x))
\end{array}\right]^{\top} P\left[\begin{array}{c}
x \\
\mathrm{dz}(u(x))
\end{array}\right],
\end{aligned}
$$

where $\operatorname{dz}(u(x))$ is the solution to (3). As in [4], we do not need to explicitly compute the mapping $x \mapsto u(x)$ because the Lyapunov construction follows from the implicit equation (3).

Due the fact that there are points where $V$ in (11) is not differentiable, it is useful to emphasize that the Lipschitz nature of $V$ allows to ignore those areas when characterizing 
local or global exponential stability of the origin. This fact is well formalized in the next lemma, which is a direct consequence of the results in [3] (see also [25]).

Lemma 1: Consider dynamics (4) and assume that there exists a locally Lipschitz Lyapunov function $x \mapsto W(x)$, positive scalars $\beta_{1}, \beta_{2}$ and $\beta_{3}$ and an open sub-level set $S(W, r):=\left\{x \in \mathbb{R}^{n}: W(x)<r\right\}$ of $W$, for some $r>0$ satisfying

$$
\begin{aligned}
& \beta_{1}|x|^{2} \leq W(x) \leq \beta_{2}|x|^{2}, \forall x \in S(W, r) \\
& \dot{W}(x):=\langle\nabla W(x), A x+B \operatorname{sat}(u(x))\rangle \leq-\beta_{3}|x|^{2},
\end{aligned}
$$

for almost all $x \in S(W, r)$,

then the origin is locally exponentially stable for (4) with basin of attraction containing $S(W, r)$. Moreover, if (12), (13) hold for any $r>0$ with the same scalars $\beta_{i}, i=1,2,3$, then the origin is globally exponentially stable for (4).

The following lemma uses Fact 3 to establish sufficient conditions for positive definiteness and radial unboundedness of $V$ in (11), while not imposing positive definiteness of matrix $P$.

Lemma 2: Given $V$ in (11) with $u$ defined in (3), if the algebraic loop in (3) is well-posed and there exist matrices $P_{11} \in \mathbb{S}_{>0}^{n}, P_{12} \in \mathbb{R}^{n \times m}, P_{22} \in \mathbb{S}^{m}$ and $T_{0} \in \mathbb{D}_{\geq 0}^{m}$ such that

$$
\Phi_{0}=\left[\begin{array}{cc}
P_{11} & P_{12}-K_{1}^{\top} T_{0} \\
P_{12}^{\top}-T_{0} K_{1} & P_{22}-T_{0} K_{2}-K_{2}^{\top} T_{0}+2 T_{0}
\end{array}\right]>0,
$$

then there exist positive scalars $\beta_{1}$ and $\beta_{2}$ satisfying (12) for any $r>0$.

Proof. The global sector condition (5) with $T=T_{0}$ implies

$$
\begin{aligned}
V(x) & \geq V(x)-2 \mathrm{dz}(u(x))^{\top} T_{0}(u(x)-\mathrm{dz}(u(x))) \\
& =\left[\begin{array}{c}
x \\
\mathrm{dz}(u(x))
\end{array}\right]^{\top} \Phi_{0}\left[\begin{array}{c}
x \\
\mathrm{dz}(u(x))
\end{array}\right] \\
& \geq \lambda_{m}\left(\Phi_{0}\right)\left|\left[\begin{array}{c}
x \\
\mathrm{dz}(u(x))
\end{array}\right]\right|^{2} \geq \lambda_{m}\left(\Phi_{0}\right)|x|^{2},
\end{aligned}
$$

which provides the lower bound in (12) with $\beta_{1}=\lambda_{m}\left(\Phi_{0}\right)$.

To study the upper bound in (12) from well-posedness of the algebraic loop (3) and Fact 2, the globally Lipschitz property of $u$, together with $u(0)=0$, ensures the existence of $L_{u}>0$ such that $|u(x)| \leq L_{u}|x|$, therefore using $|\mathrm{dz}(u(x))| \leq|u(x)| \leq L_{u}|x|$,

$$
\begin{aligned}
V(x) & \leq \lambda_{M}\left(P_{11}\right)|x|^{2}+2\left|P_{12}\right||x||\mathrm{dz}(u(x))|+|\mathrm{dz}(u(x))|^{2}\left|P_{22}\right| \\
& \leq\left(\lambda_{M}\left(P_{11}\right)+2\left|P_{12}\right| L_{u}+\left|P_{22}\right| L_{u}^{2}\right)|x|^{2}=: \beta_{2}|x|^{2},
\end{aligned}
$$

which completes the proof.

\section{B. Advantages from using a sign indefinite $P$}

A novelty of this paper is that we do not insist on the fact that matrix $P$ in (11) be positive definite, which allows representing a broader class of Lyapunov functions as compared to previous works such as [4]. As an example, consider the following two Popov-like functions, the first one focusing on the deadzone and the second one focusing (more classically, as in [14, Ex. 7.4] and [29, $\S 2.5$ and 2.1.6]) on the saturation:

$$
\begin{gathered}
V_{\text {Dai }}(x):=x^{\top} P_{0} x+\sum_{i=1}^{m} 2 \lambda_{i} \int_{0}^{u_{i}(x)} \mathrm{dz}_{i}(s) \mathrm{d} s \\
V_{\text {Khalil }}(x):=x^{\top} P_{0} x+\sum_{i=1}^{m} 2 \lambda_{i} \int_{0}^{u_{i}(x)} \operatorname{sat}_{i}(s) \mathrm{d} s .
\end{gathered}
$$

As observed in [4], using the identity $2 \int_{0}^{u_{i}(x)} \mathrm{dz}_{i}(s) d s=$ $\mathrm{dz}_{i}^{2}\left(u_{i}(x)\right)$, the first function $V_{\text {Dai }}$ in (15) can be obtained as a particular case of $V$ in (11), by setting $P_{11}=P_{0}, P_{12}=0$, $P_{22}=\operatorname{diag}\left(\lambda_{1}, \ldots, \lambda_{m}\right)=\Lambda$ and $\lambda_{i}>0$, which provides a positive definite $P$.

However, a positive definite $P$ does not provide enough degrees of freedom for representing the second function $V_{\text {Khali }}$ in (16), which can be instead represented by a sign-indefinite $P$, as follows, with the last line inspired by (11),

$$
\begin{aligned}
V_{\text {Khalil }}(x) & =x^{\top} P_{0} x+\sum_{i=1}^{m} 2 \lambda_{i} \int_{0}^{u_{i}(x)} s-\mathrm{dz}_{i}(s) \mathrm{d} s \\
& =x^{\top} P_{0} x+\sum_{i=1}^{m} \lambda_{i}\left(u_{i}(x)^{2}-\mathrm{dz}_{i}^{2}\left(u_{i}(x)\right)\right), \\
& =\left[\begin{array}{c}
x \\
\operatorname{dz}(u(x))
\end{array}\right]^{\top}\left[\begin{array}{cc}
P_{11} & P_{12} \\
P_{12}^{\top} & P_{22}
\end{array}\right]\left[\begin{array}{c}
x \\
\mathrm{dz}(u(x))
\end{array}\right] .
\end{aligned}
$$

Using the expression of $u(x)$ in (3), we obtain the following selection of $P$ in (17),

$$
P=\left[\begin{array}{ll}
P_{11} & P_{12} \\
P_{12}^{\top} & P_{22}
\end{array}\right]=\left[\begin{array}{cc}
P_{0} & 0 \\
0 & -\Lambda
\end{array}\right]+\left[\begin{array}{l}
K_{1}^{\top} \\
K_{2}^{\top}
\end{array}\right] \Lambda\left[\begin{array}{ll}
K_{1} & K_{2}
\end{array}\right],
$$

where $P_{22}$ is often nonpositive (e.g., when $K_{2}=0$ ) but yet satisfies the positivity conditions of Lemma 2 under mild conditions on matrix $P_{0}$ (these conditions are satisfied whenever $P_{0}>0$ but also allow for more general cases).

Proposition 1: If matrix $P_{0}+K_{1}^{\top} \Lambda K_{1}$ is positive definite, then condition (14) is satisfied for any $T_{0}>\frac{\Lambda}{2}$, namely under a well-posedness assumption, $V_{\text {Khalil }}$ in (16) satisfies (12) for all $r>0$.

Proof. With the structure of $V$ in (11), using the derivations in (17), function $V_{\text {Khalil }}$ in (16) corresponds to the selection of $P$ in (18). With this selection of $P$, matrix $\Phi_{0}$ in (14) reads

$$
\begin{aligned}
\Phi_{0}= & {\left[\begin{array}{cc}
P_{0} & 0 \\
0 & 2 T_{0}-\Lambda
\end{array}\right]+\left[\begin{array}{c}
K_{1}^{\top} \\
K_{2}^{\top}
\end{array}\right] \Lambda\left[\begin{array}{ll}
K_{1} & K_{2}
\end{array}\right] } \\
& +\operatorname{He}\left(\left[\begin{array}{c}
0 \\
-I_{m}
\end{array}\right] T_{0}\left[\begin{array}{ll}
K_{1} & K_{2}
\end{array}\right]\right) .
\end{aligned}
$$

Using the elimination lemma (see, e.g., [18]), defining bases $N_{1}$ and $N_{2}$ of the null-spaces of $\left[\begin{array}{ll}0 & -I_{m}\end{array}\right]$ and $\left[\begin{array}{ll}K_{1} & K_{2}\end{array}\right]$, respectively, it follows that $\Phi_{0}>0$ is equivalent to:

$$
\begin{aligned}
& N_{1}^{\top}\left(\left[\begin{array}{cc}
P_{0} & 0 \\
0 & 2 T_{0}-\Lambda
\end{array}\right]+\left[\begin{array}{c}
K_{1}^{\top} \\
K_{2}^{\top}
\end{array}\right] \Lambda\left[\begin{array}{ll}
K_{1} & K_{2}
\end{array}\right]\right) N_{1} \\
& \quad=P_{0}+K_{1}^{\top} \Lambda K_{1}>0, \\
& N_{2}^{\top}\left[\begin{array}{cc}
P_{0} & 0 \\
0 & 2 T_{0}-\Lambda
\end{array}\right] N_{2}>0,
\end{aligned}
$$

which are both satisfied under the stated assumptions. 


\section{Set inclusion for regional results}

As depicted in Figure 1, regional asymptotic stability should rely on a set inclusion property ensuring that the sublevel set

$$
S(W, 1):=\left\{x \in \mathbb{R}^{n}: W(x)<1\right\}
$$

of some suitable function $W$, based on function $V$ in (11), is contained in the set $\mathcal{S}_{h}$ of (6) (associated with function $h$ in (7)) so that the inequality of Fact 4 holds in $S(W, 1)$. Not insisting on positivity of function $V$ outside $\mathcal{S}_{h}$ can lead to extra degrees of freedom in the construction proposed in Section V (see for example the surface represented in Figure 4 and the corresponding discussion in Example 3). Therefore we propose selecting $W$ in (20) as

$$
W(x):= \begin{cases}\min \{V(x), 1\}, & \text { if } x \in \mathcal{S}_{h} \\ 1, & \text { otherwise. }\end{cases}
$$

Note that (21) does not lead, in general, to a continuous $W$, but it guarantees the identity

$$
S(W, 1)=S(V, 1) \cap \mathcal{S}_{h},
$$

which is useful for the Lyapunov analysis of Section V. ${ }^{1}$ The following lemma establishes sufficient conditions for (Lipschitz) continuity of $W$ and provides a tool, based on Fact 4, to guarantee bounds (12) with $r=1$.

Lemma 3: If the algebraic loop in (3) is well-posed and there exist matrices $P_{11} \in \mathbb{S}_{>0}^{n}, P_{12} \in \mathbb{R}^{n \times m}, P_{22} \in \mathbb{S}^{m}$, $H_{1} \in \mathbb{R}^{m \times n}, H_{2} \in \mathbb{R}^{m \times m}$ and $T \in \mathbb{D}_{>0}^{m}$ such that for all $i=1, \ldots, m$

$$
\begin{aligned}
\Phi_{1}= & {\left[\begin{array}{ccc}
P_{11} & P_{12} & 0 \\
P_{12}^{\top} & P_{22} & 0 \\
0 & 0 & 1
\end{array}\right] } \\
& +\operatorname{He}\left(\left[\begin{array}{ccc}
0 & \left(H_{1}^{\top}-K_{1}^{\top}\right) T & H_{1 i}^{\top} \\
0 & T H_{2}-T\left(K_{2}-I_{m}\right) & H_{2 i}^{\top} \\
0 & 0 & 0
\end{array}\right]\right)>0
\end{aligned}
$$

then defining $V$ and $W$ as in (11), (21), the following properties hold:

(i) function $W$ in (21) is Lipschitz continuous;

(ii) the set inclusion $S(W, 1) \subseteq \mathcal{S}_{h}$ holds, with $S(W, 1)$ and $\mathcal{S}_{h}$ defined in (20) and (6), respectively;

(iii) function $W$ satisfies (12) for $r=1$ and for suitable scalars $\beta_{1}$ and $\beta_{2}$.

Proof. As a first step of the proof, we apply a Schur complement to (23), ensuring that, for all $i=1, \ldots, m$,

$$
\begin{array}{r}
\bar{\Phi}_{1}:=P+\operatorname{He}\left(\left[\begin{array}{rr}
0 & \left(H_{1}^{\top}-K_{1}^{\top}\right) T \\
0 & T\left(H_{2}-K_{2}\right)+T
\end{array}\right]\right) \\
-\left[\begin{array}{l}
H_{1 i}^{\top} \\
H_{2 i}^{\top}
\end{array}\right]\left[\begin{array}{ll}
H_{1 i} & H_{2 i}
\end{array}\right]>0 .
\end{array}
$$

Then, based on (24), we can prove the following fact:

$$
h_{i}(x)^{2} \leq V(x), \quad \forall x \in \mathcal{S}_{h},
$$

${ }^{1}$ Condition (22) is especially useful in cases where $V(x)$ becomes smaller than 1 outside $\mathcal{S}_{h}$ (so that $S(V, 1)$ may possibly be an unbounded disconnected set), as in the example shown in Figure 4. indeed, for all $x \in \mathcal{S}_{h}$ we can use the sector condition of Fact 4 ensuring that

$$
\begin{aligned}
V(x)-h_{i}(x)^{2} & \geq V(x)-h_{i}(x)^{2}-\mathrm{dz}(u)^{\top} T(u-\mathrm{dz}(u)-h(x)) \\
& =\left[\begin{array}{c}
x \\
\mathrm{dz}(u)
\end{array}\right]^{\top} \bar{\Phi}_{1}\left[\begin{array}{c}
x \\
\mathrm{dz}(u)
\end{array}\right] \geq 0 .
\end{aligned}
$$

It then follows from (25) that at the boundary of $\mathcal{S}_{h}$, where $h_{i}(x)=1$ for some $i \in\{1, \ldots, m\}$, it must hold that $V(x) \geq 1$, which implies that $W(x)$ is continuous at the patching surfaces in (21), which proves item (i).

Item (ii) follows immediately from (22), ensuring that

$$
x \in S(W, 1) \Rightarrow x \in \mathcal{S}_{h} .
$$

As for item (iii), to show (12) for all $x \in S(W, 1)$, first notice that from definition (21) we have $W(x)=V(x)$ for all $x \in S(W, 1)$. Then, we may focus on function $V$, for establishing (12). In particular, for any $x \in S(W, 1)$ we may use Fact 4 to conclude once again from (26) that

$$
W(x)=V(x) \geq \lambda_{m}\left(\bar{\Phi}_{1}\right)|x|^{2}, \quad \forall x \in S(W, 1) .
$$

As for the upper bound in (12), we may proceed as in Lemma 2 using well-posedness of the algebraic loop (3).

\section{Global RESUlts}

\section{A. Global analysis}

Using function $V$ in (11), this section proposes conditions to solve Problem 1, as stated in the next theorem.

Theorem 1: Given $K_{1}$ and $K_{2}$, if there exist matrices $P_{11} \in$ $\mathbb{S}_{>0}^{n}, P_{12} \in \mathbb{R}^{n \times m}, P_{22} \in \mathbb{S}^{m}, T_{i} \in \mathbb{D}_{\geq 0}^{m} i \in\{0,1\}, T_{2} \in$ $\mathbb{D}_{>0}^{m}, T_{3} \in \mathbb{D}^{m}$ such that (14) and (28) (given at the top of the next page) hold, then the origin of (4) is globally exponentially stable.

Proof. The proof uses Lemma 1 by showing both (12) and (13) for any $x \in \mathbb{R}^{n}$.

To show (12), let us first observe that the $(3,3)$ term of $(28)$ reads $2 T_{2}-T_{2} K_{2}-K_{2}^{\top} T_{2}>0$, where $T_{2}>0$ by assumption. Therefore Fact 1 implies well-posedness of the algebraic loop (3) and, from Lemma 2, hypothesis (14) implies (12) globally (for any $r>0$ ).

To show (13), first note that

$$
\dot{V}(x)=2\left[\begin{array}{c}
x \\
\mathrm{dz}(u(x))
\end{array}\right]^{\top}\left[\begin{array}{ll}
P_{11} & P_{12} \\
P_{12}^{\top} & P_{22}
\end{array}\right]\left[\begin{array}{c}
\dot{x} \\
\dot{\mathrm{dz}}(u(x))
\end{array}\right] .
$$

Using Facts 3 and 5, we may bound $\dot{V}(x)$ for almost all $x \in$ $\mathbb{R}^{n}$ as follows:

$$
\begin{aligned}
\dot{V}(x) \leq & \dot{V}(x)+2 \mathrm{dz}(u(x))^{\top} T_{1}(u-\mathrm{dz}(u(x))) \\
& +2 \dot{\mathrm{d} z}(u(x))^{\top} T_{2}(\dot{u}(x)-\mathrm{d} z(u(x))) \\
& +2 \mathrm{dz}(u(x))^{\top} T_{3}(\dot{u}(x)-\dot{\mathrm{d} z}(u(x)))=-\eta^{\top} \mathcal{G} \eta,
\end{aligned}
$$




$$
\begin{aligned}
& \Psi_{1}:=\operatorname{He}\left(-\left[\begin{array}{cc}
P_{11} & P_{12} \\
P_{12}^{\top} & P_{22} \\
0 & 0
\end{array}\right]\left[\begin{array}{ccc}
A+B K_{1} & B\left(K_{2}-I_{m}\right) & 0 \\
0 & 0 & I_{m}
\end{array}\right]\right. \\
& \left.-\left[\begin{array}{ccc}
T_{1} K_{1}+T_{3} K_{1}\left(A+B K_{1}\right) & T_{1}\left(K_{2}-I_{m}\right)+T_{3} K_{1} B\left(K_{2}-I_{m}\right) & T_{3}\left(K_{2}-I_{m}\right) \\
T_{2} K_{1}\left(A+B K_{1}\right) & T_{2} K_{1} B\left(K_{2}-I_{m}\right) & T_{2}\left(K_{2}-I_{m}\right)
\end{array}\right]\right)>0
\end{aligned}
$$

where $\eta:=\left[\begin{array}{c}x \\ \mathrm{dz}(u(x)) \\ \dot{d z}(u(x))\end{array}\right]$ is an extended state, and matrix $\mathcal{G}$ can be easily evaluated as follows:

$$
\begin{aligned}
& \mathcal{G}=\operatorname{He}\left(-\left[\begin{array}{cc}
P_{11} & P_{12} \\
P_{12}^{\top} & P_{22} \\
0 & 0
\end{array}\right]\left[\begin{array}{ccc}
A+B K_{1} & B\left(K_{2}-I_{m}\right) & 0 \\
0 & 0 & I_{m}
\end{array}\right]\right) \\
& -\operatorname{He}\left(\left[\begin{array}{c}
0 \\
T_{1} \\
0
\end{array}\right]\left[\begin{array}{lll}
K_{1} & K_{2}-I_{m} & 0
\end{array}\right]\right) \\
& \left.-\operatorname{He}\left(\begin{array}{c}
0 \\
0 \\
T_{2}
\end{array}\right]\left[\begin{array}{lll}
K_{1}\left(A+B K_{1}\right) & K_{1} B\left(K_{2}-I_{m}\right) & K_{2}-I_{m}
\end{array}\right]\right) \\
& \left.-\mathrm{He}\left(\begin{array}{c}
0 \\
T_{3} \\
0
\end{array}\right]\left[\begin{array}{lll}
K_{1}\left(A+B K_{1}\right) & K_{1} B\left(K_{2}-I_{m}\right) & K_{2}-I_{m}
\end{array}\right]\right) .
\end{aligned}
$$

It is straightforward to check that the above expression corresponds to the matrix in (28), which then implies $\mathcal{G}>0$, and by (30) also implies (13) for almost all $x \in \mathbb{R}^{n}$ and completes the proof.

For the analysis result of Theorem 1 , since $K_{1}$ and $K_{2}$ are a priori given, the inequalities (14) and (28) are linear in $P_{11}, P_{12}, P_{22}$ and $T_{i}, i=0, \ldots, 3$, therefore they can be efficiently solved by a linear matrix inequality (LMI) solver (as a feasibility Semi-Definite Program).

Example 1: We illustrate here the reduced conservativeness of the conditions of Theorem 1, as compared to the use of quadratic Lyapunov functions. For global exponential stabilisation of the dynamics presented in [31, Example 4.3.1], one may attempt using static anti-windup gains and quadratic Lyapunov certificates. Unfortunately, as discussed in [31], these certificates fail to satisfy the necessary conditions for quadratic stability analysis and synthesis. It turns out that even with the anti-windup gains set to zero (mere stability analysis), the LMI conditions of our Theorem 1 ensure GES of the origin.

More specifically, the dynamics in [31, Example 4.3.1] before anti-windup corresponds to closed loop (4) with selections

$$
\begin{array}{ll}
A=\left[\begin{array}{cc}
-0.05 & 1 \\
-10 & -0.5
\end{array}\right] ; & B=\left[\begin{array}{l}
0 \\
1
\end{array}\right] ; \\
K_{1}=\left[\begin{array}{cc}
9.9 & 0.495
\end{array}\right] ; & K_{2}=0 .
\end{array}
$$

By solving LMIs (14) and (28) as per Theorem 1 one obtains matrices $P$ and $T_{i}, i=0,1,2,3$ certifying global exponential stability. In particular, for this example, Lyapunov function $V$ is characterized by matrix $P=\left[\begin{array}{ccc}1.0052 & -0.1815 & 0.0411 \\ -0.1815 & 8.4599 & -0.1985 \\ 0.0411 & -0.1985 & 0.7077\end{array}\right]$, with the eigenvalues $\left\{\begin{array}{lll}0.6982 & 1.0051 & 8.4694\end{array}\right\}$, which reveals that the degree of freedom of not constraining $P_{22}>0$ is not necessary for establishing GAS of the origin, in this example.

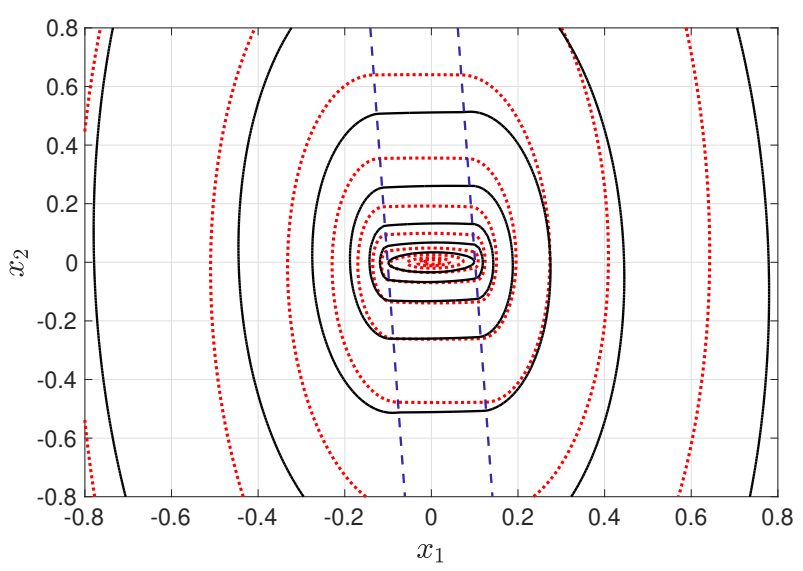

Fig. 2. Level sets of the piecewise quadratic Lyapunov function (11) (black solid). Trajectories exponentially converging to the origin (red dotted). The dashed blue lines correspond to the boundary of the set containing the origin where $\operatorname{dz}(u(x))=0$.

Figure 2 depicts in red some trajectories of the closedloop system (4). Note that the level sets of the Lyapunov function (in black) are stretched horizontally in the proximity of the origin and vertically away from the origin. This peculiar shape of the trajectories causes the infeasibility of quadratic Lyapunov conditions, well overcome by Theorem 1 .

Remark 2: The conditions of Theorem 1 readily extend to the case where dynamics (4) is generalized to

$$
\begin{aligned}
\dot{x} & =A_{\mathrm{cl}} x+B_{\mathrm{cl}} \mathrm{dz}(u(x)) \\
u(x) & =K_{1} x+K_{2} \mathrm{dz}(u(x)) .
\end{aligned}
$$

Indeed, it suffices to replace all the occurrences of $A+B K_{1}$ by $A_{\mathrm{cl}}$, and those of $B\left(K_{2}-I_{m}\right)$ by $B_{\mathrm{cl}}$ in conditions (28) and the proof of the theorem remains unchanged.

Expression (31) arises when analyzing the output feedback interconnection of a linear plant with an $n_{c}$-order dynamic controller possibly involving an anti-windup gain [23, ch. 8]:

$$
\begin{aligned}
\dot{x}_{p} & =A_{p} x_{p}+B_{p} \operatorname{sat}\left(y_{c}\right), \quad y_{p}=C_{p} x_{p} \\
\dot{x}_{c} & =A_{c} x_{c}+B_{c} y_{p}+E_{c} \mathrm{dz}\left(y_{c}\right) \\
y_{c} & =C_{c} x_{c}+D_{c} y_{p}+F_{c} \mathrm{dz}\left(y_{c}\right),
\end{aligned}
$$

which is represented by (31) with

$$
\begin{aligned}
{\left[A_{\mathrm{cl}} \mid B_{\mathrm{cl}}\right] } & =\left[\begin{array}{cc|c}
A_{p}+B_{p} D_{c} C_{p} & B_{p} C_{p} & -B_{p}\left(F_{c}-I_{m}\right) \\
B_{c} C_{p} & A_{c} & -E_{c}
\end{array}\right] \\
{\left[K_{1} \mid K_{2}\right] } & =\left[\begin{array}{cc|c}
D_{c} C_{p} & C_{c} \mid F_{c}
\end{array}\right] .
\end{aligned}
$$




\section{B. Global synthesis}

Using function $V$ in (11), we propose here conditions solving Problem 2, that is designing gains $K_{1}$ and $K_{2}$ ensuring global exponential stability of the origin.

Theorem 2: If there exist matrices $Q_{11} \in \mathbb{S}_{>0}^{n}, Q_{12} \in$ $\mathbb{R}^{n \times m}, Q_{22} \in \mathbb{S}^{m}, S \in \mathbb{D}_{>0}^{m}, M \in \mathbb{R}^{n \times n}, Y_{1} \in \mathbb{R}^{m \times n}$ and $Y_{2} \in \mathbb{R}^{m \times m}$ such that

$$
\begin{aligned}
& \Phi_{2}:=\left[\begin{array}{cc}
Q_{11} & Q_{12}-Y_{1}^{\top} \\
Q_{12}^{\top}-Y_{1} & Q_{22}-Y_{2}-Y_{2}^{\top}+2 S
\end{array}\right]>0 \\
& \Psi_{2}:=\operatorname{He}\left(-\left[\begin{array}{cc}
Q_{11} & Q_{12} \\
Q_{12}^{\top} & Q_{22} \\
0 & 0 \\
0 & 0
\end{array}\right]\left[\begin{array}{cccc}
0 & 0 & I_{n} & 0 \\
0 & 0 & 0 & I_{m}
\end{array}\right]\right. \\
& \left.-\left[\begin{array}{cccc}
A M+B Y_{1} & B Y_{2}-B S & -M & 0 \\
Y_{1} & Y_{2}-S & Y_{1} & Y_{2}-S \\
A M+B Y_{1} & B Y_{2}-B S & -M & 0 \\
0 & 0 & Y_{1} & Y_{2}-S
\end{array}\right]\right)>0
\end{aligned}
$$

then $M$ is nonsingular and the origin of system (4) with

$$
K_{1}=Y_{1} M^{-1}, \quad K_{2}=Y_{2} S^{-1},
$$

is globally exponentially stable.

Proof. First note that $M$ is nonsingular, indeed $M+M^{\top}>$ 0 is implied by the $(3,3)$ entry of $(33)$.

The rest of the proof mimics the one of Theorem 1 by using Lemma 1. In particular, to show (12), consider the change of variables $K_{1} M=Y_{1}$ and $K_{2} S=Y_{2}$ and note that inequality (32) coincides with (14) pre- and post-multiplied by $\operatorname{diag}\left(M^{\top}, S\right)$ and $\operatorname{diag}(M, S)$, respectively. Moreover, the $(4,4)$ term in (33) with $Y_{2}=K_{2} S$ reads $2 S-K_{2} S-S K_{2}^{\top}>0$, which implies well-posedness of (3) from Fact 1. Then, (12) is guaranteed by Lemma 2 .

The rest of the proof focuses on showing (13) by exploiting Fact 5 in addition to considering an enlarged space (comprising $x$ and $\dot{x}$ as in [5], [18]) so that the Lyapunov certificate is suitably separated from the dynamics matrices.

More specifically, consider the extended vector $\eta:=$

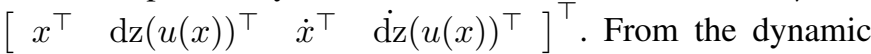
equation (4) we get, for any matrix $N$,

$$
\eta^{\top} N\left(-\dot{x}+\left(A+B K_{1}\right) x+B\left(K_{2}-I_{m}\right) \mathrm{dz}(u(x))\right)=0,
$$

which may be evaluated with $N=$ $\left[\begin{array}{cccc}M^{-\top} & 0 & M^{-\top} & 0\end{array}\right]^{\top}$, where $M \in \mathbb{R}^{n \times n}$. To establish bound (13) we may write the following bounds originating from Facts 3 and 5 evaluated with $T=S^{-1}$, and from (35):

$$
\begin{aligned}
\dot{V}(x) \leq & \dot{V}(x)+2 \mathrm{dz}(u(x))^{\top} S^{-1}(u-\mathrm{dz}(u(x))) \\
& +2 \dot{\mathrm{d} z}(u(x))^{\top} S^{-1}(\dot{u}(x)-\dot{\mathrm{d} z}(u(x))) \\
& +2 \mathrm{dz}(u(x))^{\top} S^{-1}(\dot{u}(x)-\dot{\mathrm{d} z}(u(x))) \\
& +2 \eta^{\top} N\left(-\dot{x}+\left(A+B K_{1}\right) x+B\left(K_{2}-I_{m}\right) \mathrm{dz}(u(x))\right) \\
= & -\eta^{T} \mathcal{G}_{d} \eta, \quad \text { for almost all } x \in \mathbb{R}^{n} .
\end{aligned}
$$

Thus exponential stability can be established by proving $\mathcal{G}_{d}>0$, which would prove a negative upper bound on $\dot{V}(x)$, quadratic in $x$ (and other variables too). To show that $\mathcal{G}_{d}>0$, let us equivalently study the sign of matrix

$$
\mathcal{G}_{1}:=\operatorname{diag}\left(M^{\top}, S, M^{\top}, S\right) \mathcal{G}_{d} \operatorname{diag}(M, S, M, S),
$$

and consider the change of variables $Q_{11}=M^{\top} P_{11} M, Q_{12}=$ $M^{\top} P_{12} S$ and $Q_{22}=S P_{22} S, K_{1} M=Y_{1}$ and $K_{2} S=Y_{2}$, so that the expression $\mathcal{G}_{1}$ can be written as:

$$
\begin{aligned}
\mathcal{G}_{1}= & \operatorname{He}\left(-\left[\begin{array}{cc}
Q_{11} & Q_{12} \\
Q_{12}^{\top} & Q_{22} \\
0 & 0 \\
0 & 0
\end{array}\right]\left[\begin{array}{cccc}
0 & 0 & I_{n} & 0 \\
0 & 0 & 0 & I_{m}
\end{array}\right]\right) \\
& -\operatorname{He}\left(\left[\begin{array}{c}
0 \\
I_{m} \\
0 \\
0
\end{array}\right]\left[\begin{array}{llll}
Y_{1} & Y_{2}-S & 0 & 0
\end{array}\right]\right) \\
& -\operatorname{He}\left(\left[\begin{array}{c}
0 \\
0 \\
0 \\
I_{m}
\end{array}\right]\left[\begin{array}{llll}
0 & 0 & Y_{1} & Y_{2}-S
\end{array}\right]\right) \\
& -\operatorname{He}\left(\left[\begin{array}{c}
0 \\
I_{m} \\
0 \\
0
\end{array}\right]\left[\begin{array}{llll}
0 & 0 & Y_{1} & Y_{2}-S
\end{array}\right]\right) \\
& -\operatorname{He}\left(\left[\begin{array}{c}
I_{n} \\
0 \\
I_{n} \\
0
\end{array}\right]\left[\begin{array}{llll}
A M+B Y_{1} & B Y_{2}-B S & -M & 0
\end{array}\right]\right)
\end{aligned}
$$

whose five addends clearly sum up to (33). Then, from (33) we have $\mathcal{G}_{1}>0$ and therefore $\mathcal{G}_{d}>0$, which implies, via (36), that bound (13) holds for almost all $x \in \mathbb{R}^{n}$, thus completing the proof.

Remark 3: While the analysis and synthesis results of Theorems 1 and 2 both rely on the technical tools introduced in Sections II-B and III, the extra unknowns appearing in the synthesis conditions require additional transformations and an extended space corresponding to $\eta=(x, \mathrm{dz}(u(x)), \dot{x}, \dot{\mathrm{d} z}(u(x)))$, while $\dot{x}$ does not appear in the representation of the analysis conditions. In addition to this fact, we expect the synthesis conditions to show increased conservativeness, because the coordinate transformation in the proof of Theorem 2 requires imposing $T_{1}=T_{2}=T_{3}=S^{-1}$. Due to this reason, after the gains have been selected through the synthesis conditions of Theorem 2, we expect less conservative estimates to emerge from the solution of the analysis conditions of Theorem 1. *

While the conditions of Theorem 2 ensure global exponential stability, the convergence rate of the resulting feedback law could be arbitrarily small. The next proposition provides a means to ensure that the eigenvalues of matrix $A+B K_{1}$ (namely the linear dynamics governing the tail of the converging responses) are placed in the half plane $\{s \in \mathbb{C}: \Re(s) \leq-\alpha\}$ for some desired convergence rate $\alpha>0$. We emphasize that the convergence rate $\alpha>0$, as stated in the next proposition, is a local property (related to $\left.A+B K_{1}\right)$ and therefore is ensured only in a neighborhood of the origin where the saturation is not active.

Proposition 2: Given a desired convergence rate $\alpha>0$, if there exist matrices $R \in \mathbb{S}_{>0}^{n}, Q_{11} \in \mathbb{S}_{>0}^{n}, Q_{12} \in \mathbb{R}^{n \times m}$, $Q_{22} \in \mathbb{S}^{m}, S \in \mathbb{D}_{>0}^{m}, M \in \mathbb{R}^{n \times n}, Y_{1} \in \mathbb{R}^{m \times n}$ and $Y_{2} \in$ $\mathbb{R}^{m \times m}$ satisfying (32), (33) and

$$
R=R^{\top}>0, \operatorname{He}\left[\begin{array}{cc}
\alpha M+A M+B Y_{1} & -M \\
\alpha M+A M+B Y_{1}+R & -M
\end{array}\right]<0,
$$


then matrix $M$ is nonsingular, the origin of system (4) with selections (34) is globally exponentially stable, and the eigenvalues of $A+B K_{1}$ have real part smaller than $-\alpha$.

Proof. Global exponential stability has been already proven in Theorem 2. To show that the eigenvalues of $A+B K_{1}$ have real part smaller than $-\alpha$, it is enough to show that matrix $\bar{A}:=A+B K_{1}+\alpha I$ is Hurwitz. To this end, condition (37) pre-multiplied by $\operatorname{diag}\left(M^{-\top}, M^{-\top}\right)$ and post-multiplied by $\operatorname{diag}\left(M^{-1}, M^{-1}\right)$ reads

$$
\left[\begin{array}{cc}
0 & \bar{R} \\
\bar{R} & 0
\end{array}\right]+\operatorname{He}\left(\left[\begin{array}{l}
M^{-\top} \\
M^{-\top}
\end{array}\right]\left[\begin{array}{ll}
\bar{A} & -I_{n}
\end{array}\right]\right)<0
$$

with the Lyapunov certificate $\bar{R}=M^{-\top} R M^{-1} \geq 0$. Preand post-multiplying the above inequality by $\left[\frac{z}{\bar{A} z}\right]^{\top}$ and its transpose, we obtain

$$
2 z^{\top} \bar{R} \bar{A} z=2 z^{\top} \bar{R}\left(A+B K_{1}+\alpha I\right) z<0, \forall z \neq 0,
$$

which completes the proof.

\section{REgIONAL STABILITY}

In this section, we address Problems 3 and 4 following the idea of the set inclusion in Figure 1 and exploiting the result in Lemma 3. We first present analysis results and then synthesis results, paralleling the previous section.

\section{A. Regional analysis}

Using Lemma 3, the next theorem proposes conditions ensuring local exponential stability of the origin for system (4), thus providing a solution to Problem 3.

Theorem 3: Given any scalar $\tau>0$, if there exist matrices $Q_{11} \in \mathbb{S}_{>0}^{n}, Q_{12} \in \mathbb{R}^{n \times m}, Q_{22} \in \mathbb{S}^{m}, T_{1}=$ $\operatorname{diag}\left\{t_{1}, \ldots, t_{m}\right\}, T_{2} \in \mathbb{D}_{>0}^{m}, T_{3} \in \mathbb{D}^{m}, Z_{1} \in \mathbb{R}^{m \times n}$, $Z_{2} \in \mathbb{R}^{m \times m}$ such that

$$
\begin{aligned}
T_{1}> & \tau I_{m}, \\
\Phi_{3}:= & {\left[\begin{array}{ccc}
P_{11} & P_{12} & 0 \\
P_{12}^{\top} & P_{22} & 0 \\
0 & 0 & \tau t_{i}
\end{array}\right] } \\
& +\operatorname{He}\left[\begin{array}{ccc}
0 & Z_{1}^{\top}-K_{1}^{\top} T_{1} & Z_{1 i}^{\top} \\
0 & Z_{2}-T_{1}\left(K_{2}-I_{m}\right) & Z_{2 i}^{\top} \\
0 & 0 & 0
\end{array}\right]>0,
\end{aligned}
$$

for all $i=1, \ldots, m$, and condition (40) (given at the top of the next page) holds, then the origin is locally exponentially stable for closed loop (4) and its basin of attraction contains set $S(W, 1)$ defined in (20), with functions $V$ and $W$ in (11), (21) characterized by $P=\left[\begin{array}{ll}P_{11} & P_{12} \\ P_{12}^{\top} & P_{22}\end{array}\right]$.

Proof. The proof relies on Lemma 1 by showing the two bounds (12) and (13) with $r=1$.

To show (12), let us first observe that the $(3,3)$ term of $(40)$ reads $2 T_{2}-T_{2} K_{2}-K_{2}^{\top} T_{2}>0$, where $T_{2}>0$ by assumption, so that Fact 1 implies well-posedness of the algebraic loop (3). Moreover, defining $H_{1}=T_{1}^{-1} Z_{1}$ and $H_{2}=T_{1}^{-1} Z_{2}$ and combining (38) with (39), we get

$$
\begin{aligned}
\bar{\Phi}_{3} & :=\left[\begin{array}{ccc}
P_{11} & P_{12} & 0 \\
P_{12}^{\top} & P_{22} & 0 \\
0 & 0 & t_{i}^{2}
\end{array}\right]+\text { He }\left[\begin{array}{ccc}
0 & Z_{1}^{\top}-K_{1}^{\top} T_{1} & Z_{1 i}^{\top} \\
0 & Z_{2}-T_{1} K_{2}+T_{1} & Z_{2 i}^{\top} \\
0 & 0 & 0
\end{array}\right] \\
& >\Phi_{3}>0 .
\end{aligned}
$$

Left and right multiplying $\bar{\Phi}_{3}>0$ by $\operatorname{diag}\left(I_{n}, I_{m}, t_{i}^{-1}\right)$, we obtain (23) so that all the assumptions of Lemma 3 hold. Therefore, from item (i), function $W$ in (21) is Lipschitz continuous and coincides with $V$ (of (11) in the open set $S(W, 1)$ ). Moreover, from item (iii), bound (12) holds.

To show (13), first consider item (ii) of Lemma 3 ensuring that for all $x \in S(W, 1)$ we have $x \in \mathcal{S}_{h}$ and we may use the local condition (8) of Fact 4 . Then proceeding as in the proof of Theorem 1 but using Fact 4 in place of Fact 3 (because we focus on local results), using expressions (29), we get for almost all $x \in \mathbb{R}^{n}$

$$
\begin{aligned}
\dot{W}(x) \leq & \dot{W}(x)+2 \mathrm{dz}(u(x))^{\top} T_{1}(u-\mathrm{dz}(u(x))-h(x)) \\
& +2 \dot{\mathrm{d} z}(u(x))^{\top} T_{2}(\dot{u}(x)-\dot{\mathrm{d} z}(u(x))) \\
& +2 \mathrm{dz}(u(x))^{\top} T_{3}(\dot{u}(x)-\dot{\mathrm{d} z}(u(x)))=-\eta^{\top} \mathcal{G}_{l} \eta
\end{aligned}
$$

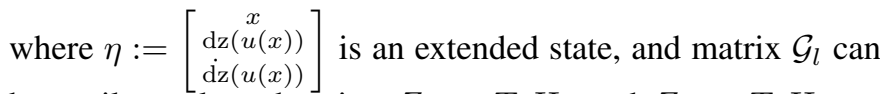
be easily evaluated, using $Z_{1}=T_{1} H_{1}$ and $Z_{2}=T_{1} H_{2}$, as follows:

$$
\begin{aligned}
& \mathcal{G}_{l}=\operatorname{He}\left(-\left[\begin{array}{cc}
P_{11} & P_{12} \\
P_{12}^{\top} & P_{22} \\
0 & 0
\end{array}\right]\left[\begin{array}{ccc}
A+B K_{1} & B\left(K_{2}-I_{m}\right) & 0 \\
0 & 0 & I_{m}
\end{array}\right]\right) \\
& -\mathrm{He}\left(\left[\begin{array}{c}
0 \\
I_{m} \\
0
\end{array}\right]\left[\begin{array}{lll}
T_{1} K_{1}-Z_{1} & T_{1}\left(K_{2}-I_{m}\right)-Z_{2} & 0
\end{array}\right]\right) \\
& \left.-\mathrm{He}\left(\begin{array}{c}
0 \\
0 \\
T_{2}
\end{array}\right]\left[\begin{array}{lll}
K_{1}\left(A+B K_{1}\right) & K_{1} B\left(K_{2}-I_{m}\right) & K_{2}-I_{m}
\end{array}\right]\right) \\
& -\mathrm{He}\left(\left[\begin{array}{c}
0 \\
T_{3} \\
0
\end{array}\right]\left[\begin{array}{lll}
K_{1}\left(A+B K_{1}\right) & K_{1} B\left(K_{2}-I_{m}\right) & K_{2}-I_{m}
\end{array}\right]\right) .
\end{aligned}
$$

It is straightforward to check that the above expression corresponds to the matrix in (40), which then implies $\mathcal{G}_{l}>0$, and by (41) implies (13) for almost all $x \in S(W, 1)$ thus completing the proof.

Since the $\mathrm{dz}$ function is zero around the origin, a necessary and sufficient condition for local asymptotic (and exponential) stability of the origin for closed loop (4) is that matrix $A+B K_{1}$ is Hurwitz. The advantage of using more advanced conditions, such as those of Theorem 3 , is that of obtaining nontrivial estimates $S(W, 1)$ of the basin of attraction. The next proposition provides a useful means for maximizing the size of this estimate by guaranteeing the inclusion

$$
\mathcal{E}(\hat{P}, 1):=\left\{x \in \mathbb{R}^{n}: x^{\top} \hat{P} x<1\right\} \subset S(W, 1),
$$

so that minimizing $\hat{P}$ in some suitable way ensures enlarged estimates $S(W, 1)$.

Proposition 3: Given any scalar $\tau>0$, if there exist matrices $\hat{P} \in \mathbb{S}_{>0}^{n}, \hat{T} \in \mathbb{D}_{>0}^{m}, Q_{11} \in \mathbb{S}_{>0}^{n}, Q_{12} \in \mathbb{R}^{n \times m}$, $Q_{22} \in \mathbb{S}^{m}, T_{1}=\operatorname{diag}\left\{t_{1}, \ldots, t_{m}\right\}, T_{2} \in \mathbb{D}_{>0}^{m}, T_{3} \in \mathbb{D}^{m}$, $Z_{1} \in \mathbb{R}^{m \times n}, Z_{2} \in \mathbb{R}^{m \times m}$ satisfying (38), (39), (40) and

$$
\frac{1}{2} \mathrm{He}\left[\begin{array}{cc}
\hat{P}-P_{11} & -2\left(P_{12}+K_{1}^{\top} \hat{T}\right) \\
0 & -P_{22}-\left(K_{2}-I_{m}\right)^{\top} \hat{T}-\hat{T}\left(K_{2}-I_{m}\right)
\end{array}\right]>0,
$$

then the origin is locally exponentially stable for closed loop (4) and its basin of attraction contains set $S(W, 1)$ defined in (20), which satisfies inclusion (42). 


$$
\begin{aligned}
& \Psi_{3}:=\operatorname{He}\left(-\left[\begin{array}{cc}
P_{11} & P_{12} \\
P_{12}^{\top} & P_{22} \\
0 & 0
\end{array}\right]\left[\begin{array}{ccc}
A+B K_{1} & B\left(K_{2}-I_{m}\right) & 0 \\
0 & 0 & I_{m}
\end{array}\right]\right. \\
& 0 \\
& \left.-\left[\begin{array}{ccc}
T_{1} K_{1}-Z_{1}+T_{3} K_{1}\left(A+B K_{1}\right) & T_{1}\left(K_{2}-I_{m}\right)-Z_{2}+T_{3} K_{1} B\left(K_{2}-I_{m}\right) & T_{3}\left(K_{2}-I_{m}\right) \\
T_{2} K_{1}\left(A+B K_{1}\right) & T_{2} K_{1} B\left(K_{2}-I_{m}\right) & T_{2}\left(K_{2}-I_{m}\right)
\end{array}\right]\right)>0
\end{aligned}
$$
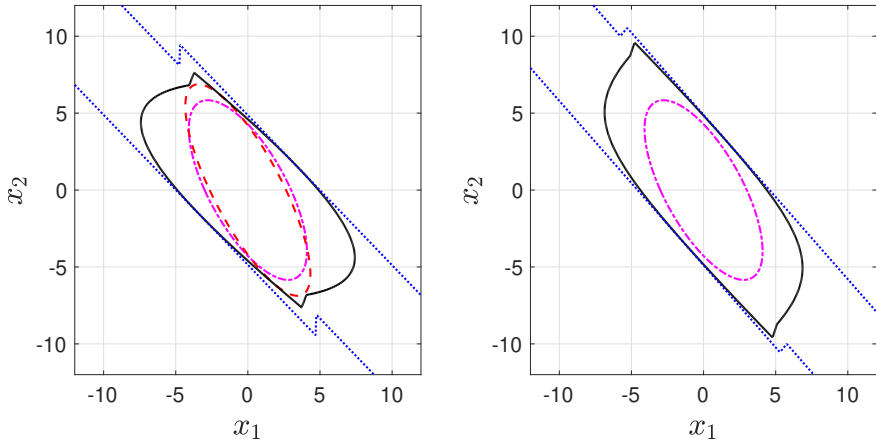

Fig. 3. Example 2: the left figure shows the set $S(W, 1)$ (in black) obtained when solving (44) and the maximized ellipsoid $\mathcal{E}(\hat{P}, 1) \subset S(W, 1)$ (dashed red ellipse). On the right we see the set $S(W, 1)$ obtained when minimizing the trace of $P_{11}$.

Proof. The proof of local exponential stability with $S(W, 1)$ contained in the basin of attraction has been already given in Theorem 3. We prove below that the additional LMI (43) ensures (42). To this end, it is enough to show that $\left(x^{\top} \hat{P} x<\right.$ $1) \Rightarrow(S(W, 1)<1)$, which holds if we show that

$$
x^{\top} \hat{P} x>V(x), \quad \forall x \in \mathcal{S}_{h} .
$$

By using the global sector condition in (5) with $T=\hat{T}$, the inequality above is ensured if

$$
x^{\top} \hat{P} x-V(x)-\mathrm{dz}(u)^{\top} \hat{T}(u-\mathrm{d} z(u))>0,
$$

which can be checked to be a quadratic form in $\left[\begin{array}{c}x \\ \mathrm{dz}(u(x))\end{array}\right]$ involving the positive definite matrix in (44), thus completing the proof.

Remark 4: As expressed in Theorem 3 and its proof, the trick used to handle $t_{i}^{2}$ which appears in $\bar{\Phi}_{3}$ consists in replacing it by $\tau t_{i}$, where $\tau$ is upper bounded by $T_{1}$. Moreover, $\tau$ may be either a scalar (as suggested in Theorem 3 ) or, when the dimension of the input is small, may be set as a vector, thus allowing us to replace (39) by $t_{i}>\tau_{i}$, for all $i=1, \ldots, m$. The selection of a suitable $\tau$ may be performed either with a simple grid search or cast in a nonlinear optimization procedure (such as the fminsearch Matlab function) including a convex optimization problem based on Proposition 3, corresponding to

$$
\begin{aligned}
& \min \operatorname{Tr}(\hat{P}) \text {, subject to: }
\end{aligned}
$$

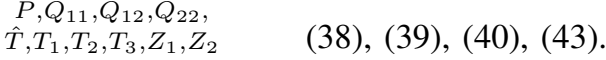

The objective function given by the trace of $\hat{P}$ is used as an indication of the size of the estimate of the basin of attraction. Example 2 reported below illustrates the use of this optimization criterion.
Example 2: Consider the load balancing example reported in [23, Ex. 1.1] corresponding to the closed loop (4) with:

$$
A=\left[\begin{array}{ll}
0 & 1 \\
1 & 0
\end{array}\right], B=\left[\begin{array}{c}
0 \\
-5
\end{array}\right], K_{1}=\left[\begin{array}{ll}
2.6 & 1.4
\end{array}\right], K_{2}=0 .
$$

For this example, we first solve the conditions of Theorem 3 and Proposition 3 adopting the optimization strategy proposed in Remark 4 and minimizing the trace of matrix $\hat{P}$ as in (44). The resulting level set $S(W, 1)$ is shown in black at the left of Figure 3 (already reported in Figure 1). The boundary of the set $\mathcal{E}(\hat{P}, 1)$ is shown in dashed red and is contained in $S(W, 1)$, as proven in Proposition 3. The dashed-dotted magenta ellipse in the same figure corresponds to a quadratic estimate of the basin of attraction obtained with the analysis results in [23, Prop. 3.1], illustrating the increased ability of providing improved estimates of the basin of attraction with sign-indefinite quadratic forms and Theorem 3. The dotted blue lines denote the boundaries of set $\mathcal{S}_{h}$ in (6), where the local sector condition (8) is valid (and where function $W$ coincides with $V$ ). The peculiar shape of these boundaries is obtained thanks to the term $H_{2} \mathrm{dz}(u(x))$ in (7).

The black level set $S(W, 1)$ shown at the right of Figure 3 corresponds to replacing problem (44) by a heuristic approach minimizing the trace of $P_{11}$ (the upper left subentry of $P$ associated to vector $x$, as per (11)) under constraints (38)-(40). While this criterion only indirectly addresses the maximization of the set $S(W, 1)$, the right plot indicates that it leads to a slightly larger set $S(W, 1)$, as compared to the solution proposed in Proposition 3. In both cases the obtained estimates are significantly larger than the quadratic estimates obtained with [23, Prop. 3.1] (dashed-dotted magenta).

For this example, the optimization of Remark 4 returns a sign-indefinite solution $P$ corresponding to

$$
P=\left[\begin{array}{ccc}
0.0428 & 0.0030 & 0.0833 \\
0.0030 & 0.0100 & 0.0570 \\
0.0833 & 0.0570 & -0.0697
\end{array}\right]
$$

where we can see that $P_{22}=-0.0697$ is negative.

Example 3: We present an example showing that the Lyapunov-like function $V$ may become negative outside $\mathcal{S}_{h}$, which motivates introducing function $W$ in (21), because $S(V, 1)$ is an unbounded set, while $S(W, 1)$ is bounded and contained in $\mathcal{S}_{h}$. Figure 4 reports the surface of $V$ and shows in black the level set $V(x)=1$. Function $V$ is obtained for the exponentially unstable plant (4) with:

$$
A=\left[\begin{array}{ll}
0 & 1 \\
0 & 1
\end{array}\right], B=\left[\begin{array}{c}
-1 \\
1
\end{array}\right], K_{1}=\left[\begin{array}{ll}
-0.1 & 2
\end{array}\right], K_{2}=0.9,
$$

by solving (38)-(40) while minimizing the trace of matrix $P_{11}$, as in the right picture in Figure 3 . Note that $S(V, 1)$ 


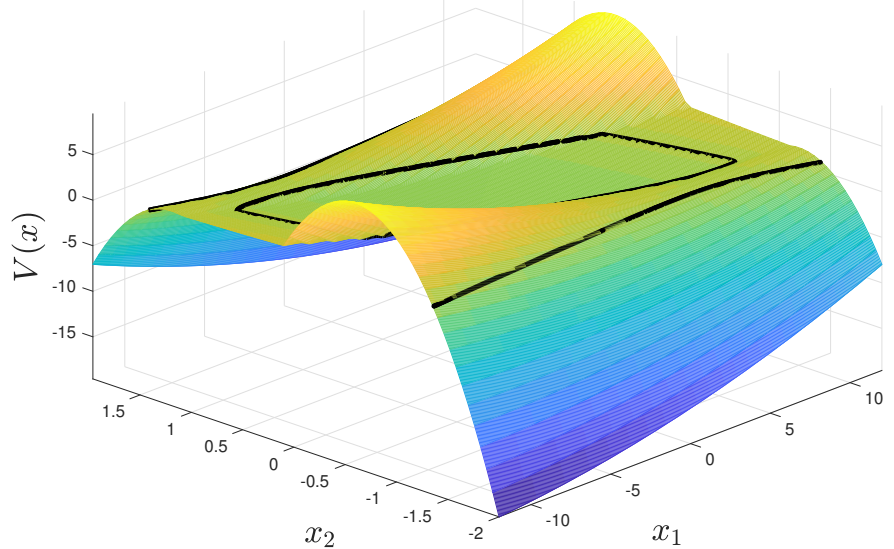

Fig. 4. Example 3: surface of the Lyapunov-like function $V(x)$ and the sub-level corresponding to $V(x)=1$ (in black).

is a disconnected unbounded set (unbounded in directions where $x_{2}$ grows unbounded) while $S(W, 1)$ only corresponds to the inner square-shaped connected set. Also in this case, the optimal value of $P$ is not sign definite and corresponds to

$$
P=\left[\begin{array}{ccc}
0.0152 & 0.0264 & -0.4719 \\
0.0264 & 0.1066 & -5.8609 \\
-0.4719 & -5.8609 & -9.6106
\end{array}\right]
$$

Based on Remark 1, we may numerically investigate the robustness of stability when the artificial saturation level $u_{\max }=1$ embedded in the controller is different from the actual physical saturation level $u_{\text {max,ph }}$ trimming the plant input. Clearly, only the cases where $u_{\max , \mathrm{ph}} \leq u_{\max }=1$ are interesting because the case $u_{\max , \mathrm{ph}} \geq u_{\max }$ does not pose any problem. We characterize three cases below, by picking an initial condition close to the boundary of $S(W, 1)$ and perturbing the initial condition and the physical saturation level:

- Case 1. Consider the initial condition $x_{0}=\left[\begin{array}{ll}4 & -0.82\end{array}\right]^{\top}$ belonging to the guaranteed stability set $S(W, 1)$ (indeed $\left.V\left(x_{0}\right)=0.9781<1\right)$. When shrinking the saturation level up to $u_{\text {max,ph }}=0.8205$ the closed-loop trajectories preserve convergence the origin. With $u_{\text {max,ph }}=0.8200$, the closedloop trajectory diverges.

- Case 2. Consider the initial condition $x_{0}=\left[\begin{array}{ll}4 & -0.95\end{array}\right]^{\top}$, which does not belong to $S(W, 1)$ (indeed $V\left(x_{0}\right)=1.431>$ $1)$. The closed-loop trajectory converges to zero with $u_{\text {max,ph }}=$ 1 but diverges with $u_{\text {max,ph }}=0.95$.

- Case 3. Consider the initial condition $x_{0}=\left[\begin{array}{ll}4 & -1\end{array}\right]^{\top}$, which does not belong to $S(W, 1)$ (indeed $V\left(x_{0}\right)=1.435>$ 1 ). The closed-loop solution already diverges with $u_{\text {max,ph }}=1$.

Both Cases 1 and 2 show some level of robustness with uncertain saturation levels, whereas Case 3 show that the estimate of the basin of attraction is not too conservative. 0

Remark 5: Similar to the observations in Remark 2 for the global case, the analysis conditions in Theorem 3 and the optimization problem in (44) readily extend to the case where dynamics (4) is replaced by the more general form (31) and may be used to address output feedback dynamic controllers with anti-windup action.

\section{B. Regional synthesis}

The following theorem provides conditions to solve Problem 4 by suitably selecting feedback gains $K_{1}, K_{2}$.

Theorem 4: If there exist matrices $Q_{11} \in \mathbb{S}_{>0}^{n}, Q_{12} \in$ $\mathbb{R}^{n \times m}, Q_{22} \in \mathbb{S}^{m}, M \in \mathbb{R}^{n \times n}, S \in \mathbb{D}_{>0}^{m}, Z_{1} \in \mathbb{R}^{m \times n}$, $Z_{2} \in \mathbb{R}^{m \times m}, Y_{1} \in \mathbb{R}^{m \times n}, Y_{2} \in \mathbb{R}^{m \times m}$ such that

$\Phi_{4}:=\left[\begin{array}{ccc}Q_{11} & Q_{12} & 0 \\ Q_{12}^{\top} & Q_{22} & 0 \\ 0 & 0 & 1\end{array}\right]+\operatorname{He}\left[\begin{array}{ccc}0 & Z_{1}^{\top}-Y_{1}^{\top} & Z_{1 i}^{\top} \\ 0 & Z_{2}-Y_{2}+S & Z_{2 i}^{\top} \\ 0 & 0 & 0\end{array}\right]>0$

and condition (46) (given at the top of next page) hold then matrix $M$ is nonsingular. Moreover, the origin is locally exponentially stable for the closed loop (4) with $K_{1}$ and $K_{2}$ chosen as in (34), and its basin of attraction contains the set $S(W, 1)$ defined in (20), with functions $V$ and $W$ in (11), (21) characterized by

$$
P=\left[\begin{array}{cc}
M^{-\top} & 0 \\
0 & S^{-1}
\end{array}\right]\left[\begin{array}{ll}
Q_{11} & Q_{12} \\
Q_{12}^{\top} & Q_{22}
\end{array}\right]\left[\begin{array}{cc}
M^{-1} & 0 \\
0 & S^{-1}
\end{array}\right]
$$

Proof. We first show that $M$ is nonsingular, which is a direct consequence of $M+M^{\top}>0$ implied by the $(3,3)$ entry of $\Psi_{4}$ in (46). The rest of the proof relies on Lemma 1, by showing the two bounds (12) and (13) with $r=1$.

To show (12), let us first observe that with $Y_{2}=K_{2} S$. the $(4,4)$ term of $(46)$ reads $2 S-S K_{2}-K_{2}^{\top} S>0$, where $S>0$ by assumption, so that Fact 1 implies well-posedness of the algebraic loop (3). Moreover, left and right multiplying $\Phi_{4}>0$ by $\operatorname{diag}\left(M^{\top}, S, 1\right)$ and $\operatorname{diag}(M, S, 1)$, respectively, and also using $Y_{1}=K_{1} M$ and $Y_{2}=K_{2} S$ and $P$ as in (47), we obtain $\Phi_{1}>0$, with $\Phi_{1}$ as in (23). As a consequence, Lemma 3 holds and function $W$ in (21) is Lipschitz continuous (item (i)) and satisfies (12) with $r=1$ (item (iii)).

To show (13), item (ii) of Lemma 3 ensures $S(W, 1) \subset \mathcal{S}_{h}$, which allows applying the local conditions (8) of Fact 4 to all $x \in S(W, 1)$. In particular, we exploit Facts 4 and 5 evaluated with $T=S^{-1}$ and the change of variables

$$
\begin{array}{ll}
Z_{1}=H_{1} M, & Z_{2}=H_{2} S \\
Q_{11}=M^{\top} P_{11} M, & Q_{12}=M^{\top} P_{12} S, \quad Q_{22}=S P_{22} S,
\end{array}
$$

following similar steps to the proof of Theorem 2 on an extended space parametrized by $\eta:=$

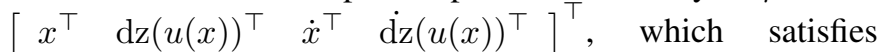
bound (35). Recalling that $W(x)=V(x)$ for all $x$ in the open set $S(W, 1)$, we have

$$
\begin{aligned}
& \dot{W}(x) \leq \dot{W}(x)+2 \mathrm{dz}(u)^{\top} S^{-1}(u-\mathrm{dz}(u)-h(x)) \\
& \quad+2 \dot{\mathrm{d} z}(u(x))^{\top} S^{-1}(\dot{u}(x)-\dot{\mathrm{d} z}(u(x))) \\
& \quad+2 \mathrm{dz}(u(x))^{\top} S^{-1}(\dot{u}(x)-\dot{\mathrm{d} z}(u(x))) \\
& -2 \eta^{\top} N\left(-\dot{x}+\left(A+B K_{1}\right) x+B\left(K_{2}-I_{m}\right) \mathrm{dz}(u(x))\right) \\
& =-\eta^{\top} \bar{\Psi}_{4} \eta, \quad \text { for almost all } x \in S(W, 1) .
\end{aligned}
$$




$$
\Psi_{4}:=\operatorname{He}\left(-\left[\begin{array}{cc}
Q_{11} & Q_{12} \\
Q_{12}^{\top} & Q_{22} \\
0 & 0
\end{array}\right]\left[\begin{array}{cccc}
0 & 0 & I_{n} & 0 \\
0 & 0 & 0 & I_{m}
\end{array}\right]-\left[\begin{array}{cccc}
A M+B Y_{1} & B Y_{2}-B S & -M & 0 \\
Y_{1}-Z_{1} & Y_{2}-Z_{2}-S & Y_{1} & Y_{2}-S \\
A M+B Y_{1} & B Y_{2}-B S & -M & 0 \\
0 & 0 & Y_{1} & Y_{2}-S
\end{array}\right]\right)>0
$$

By selecting $N=\left[\begin{array}{llll}M^{-\top} & 0 & M^{-\top} & 0\end{array}\right]^{\top}$ in (49), and using the change of variables (48), together with $Y_{1}=K_{1} M$ and $Y_{2}=K_{2} S$, we get

$$
\begin{aligned}
& \hat{\Psi}_{4}=\operatorname{diag}\left(M^{\top}, S, M^{\top}, S\right) \bar{\Psi}_{4} \operatorname{diag}(M, S, M, S) \\
& =\operatorname{He}\left(-\left[\begin{array}{cc}
Q_{11} & Q_{12} \\
Q_{12}^{\top} & Q_{22} \\
0 & 0 \\
0 & 0
\end{array}\right]\left[\begin{array}{cccc}
0 & 0 & I_{n} & 0 \\
0 & 0 & 0 & I_{m}
\end{array}\right]\right) \\
& -\operatorname{He}\left(\left[\begin{array}{c}
0 \\
I_{m} \\
0 \\
0
\end{array}\right]\left[\begin{array}{llll}
K_{1} M-Z_{1} & K_{2} S-Z_{2}-S & 0 & 0
\end{array}\right]\right) \\
& -\operatorname{He}\left(\left[\begin{array}{c}
0 \\
0 \\
0 \\
I_{m}
\end{array}\right]\left[\begin{array}{llll}
0 & 0 & K_{1} M & K_{2} S-S
\end{array}\right]\right) \\
& -\operatorname{He}\left(\left[\begin{array}{c}
0 \\
I_{m} \\
0 \\
0
\end{array}\right]\left[\begin{array}{llll}
0 & 0 & K_{1} M & K_{2} S-S
\end{array}\right]\right) \\
& -\operatorname{He}\left(\left[\begin{array}{c}
I_{n} \\
0 \\
I_{n} \\
0
\end{array}\right]\left[\begin{array}{llll}
A M+B K_{1} M & B K_{2} S-B S & -M & 0
\end{array}\right]\right),
\end{aligned}
$$

which coincides with $\Psi_{4}$ appearing in (46), and is positive definite by hypothesis. As a consequence, $\bar{\Psi}_{4}>0$ which, by (49), implies (13) with $r=1$, thus concluding the proof.

Remark 6: From Theorems 3 and 4, one can recover the global conditions of Theorems 1 and 2 by setting $Z_{1}=0$ and $Z_{2}=0$. In particular, the observations in Remark 3 apply also to the regional case, and we expect the main source of conservativeness to come from the fact that the multipliers $T_{i}$ of the sector-like conditions are set to be the same (and equal to $S^{-1}$ ) in the synthesis LMIs, to preserve convexity.

Similar to the discussion after Theorems 2 and 3 , is it useful to provide guaranteed performance criteria that may be enforced together with the LMI conditions (45), (46) of Theorem 4. The next two propositions provide parallel results to those of Propositions 2 and 3 for the regional synthesis results of Theorem 4.

Proposition 4: Given a desired convergence rate $\alpha>0$, if there exist matrices $R \in \mathbb{S}_{>0}^{n}, Q_{11} \in \mathbb{S}_{>0}^{n}, Q_{12} \in \mathbb{R}^{n \times m}$, $Q_{22} \in \mathbb{S}^{m}, M \in \mathbb{R}^{n \times n}, S \in \mathbb{D}_{>0}^{m}, Z_{1} \in \mathbb{R}^{m \times n}, Z_{2} \in \mathbb{R}^{m \times m}$, $Y_{1} \in \mathbb{R}^{m \times n}, Y_{2} \in \mathbb{R}^{m \times m}$ satisfying (45), (46), and (37), then matrix $M$ is nonsingular. Moreover, the origin is locally exponentially stable for the closed loop (4) with $K_{1}$ and $K_{2}$ chosen as in (34), and its basin of attraction contains set $S(W, 1)$ defined in (20), with functions $V$ and $W$ in (11), (21) characterized by (47). Moreover, the eigenvalues of $A+B K_{1}$ have real part smaller than $-\alpha$.

Proof. The proof is a straightforward combination of the proof of Theorem 4 and Proposition 2.
Proposition 5: If there exist matrices $\hat{P} \in \mathbb{S}_{>0}^{n}, Q_{11} \in \mathbb{S}_{>0}^{n}$, $Q_{12} \in \mathbb{R}^{n \times m}, Q_{22} \in \mathbb{S}^{m}, M \in \mathbb{R}^{n \times n}, S \in \mathbb{D}_{>0}^{m}, Z_{1} \in \mathbb{R}^{m \times n}$, $Z_{2} \in \mathbb{R}^{m \times m}, Y_{1} \in \mathbb{R}^{m \times n}, Y_{2} \in \mathbb{R}^{m \times m}$ satisfying (45), (46), and

$$
\left[\begin{array}{ccc}
M+M^{\top}-Q_{11} & I_{n} & -Q_{12}-Y_{1}^{\top} \\
I_{n} & \hat{P} & 0 \\
-Q_{12}^{\top}-Y_{1} & 0 & -Q_{22}+2 S-Y_{2}-Y_{2}^{\top}
\end{array}\right]>0,
$$

then matrix $M$ is nonsingular. Moreover, the origin is locally exponentially stable for the closed loop (4) with $K_{1}$ and $K_{2}$ chosen as in (34). Finally, its basin of attraction contains set $S(W, 1)$ defined in (20) (with $V$ in (11) characterized by (47)), and the set $S(W, 1)$ satisfies inclusion (42).

Proof. The proof of local exponential stability of the origin with basin of attraction containing the set $S(W, 1)$ has been given in Theorem 4. We prove below that inclusion (42) holds. To this end, by Proposition 3, it is enough to show that (50) implies (43), which we do in the rest of the proof.

To prove (43) apply a Schur complement to (50) to obtain

$$
\left[\begin{array}{cc}
M+M^{\top}-\hat{P}^{-1}-Q_{11} & -Q_{12}-Y_{1}^{\top} \\
-Q_{12}^{\top}-Y_{1} & -Q_{22}+2 S-Y_{2}-Y_{2}^{\top}
\end{array}\right]>0,
$$

and then observe that $\left(\hat{P}^{-1}-M^{\top}\right) \hat{P}\left(\hat{P}^{-1}-M\right) \geq 0$ implies $M+M^{\top}-\hat{P}^{-1} \leq M^{\top} \hat{P} M$, so that

$$
\left[\begin{array}{cc}
M^{\top} \hat{P} M-Q_{11} & -Q_{12}-Y_{1}^{\top} \\
-Q_{12}^{\top}-Y_{1} & -Q_{22}+2 S-Y_{2}-Y_{2}^{\top}
\end{array}\right]>0 .
$$

Finally, pre- and post-multiplying the previous inequality by $\operatorname{diag}\left(M^{\top}, S\right)$ and $\operatorname{diag}(M, S)$, respectively, and recalling that $Y_{1}=K_{1} M$ and $Y_{2}=K_{2} S$, we obtain (43).

Remark 7: Combining the results of Propositions 4 and 5, we may optimally choose the gains $K_{1}$ and $K_{2}$ by first fixing a desired convergence rate $\alpha$, and then solving the optimization

$$
\begin{array}{ll}
\min _{\substack{R, \hat{P}, Q_{11}, Q_{12} Q_{22}, M, S, Z_{1}, Z_{2}, Y_{1}, Y_{2}}} \operatorname{Tr}(\hat{P}), \text { subject to: } \\
\text { (45), (46), (37), (50) }
\end{array}
$$

so that the size of the estimate of the basin of attraction $S(W, 1)$ is maximized by minimizing the trace of $\hat{P}$, while guaranteeing the local convergence rate $\alpha$. This type of tradeoff is well explored in Example 4 discussed below.

Example 4: Consider the longitudinal dynamics of an F8 aircraft from [28], modeled by plant (1) with matrices

$$
\begin{aligned}
& {[A \mid B]} \\
& =\left[\begin{array}{cccc|cc}
-0.8 & -0.006 & -12 & 0 & -19 & -3 \\
0 & -0.014 & -16.64 & -32.2 & -0.66 & -0.5 \\
1 & -0.0001 & -1.5 & 0 & -0.16 & -0.5 \\
1 & 0 & 0 & 0 & 0 & 0
\end{array}\right] .
\end{aligned}
$$

To design $K_{1}$ and $K_{2}$, we solve optimization (51) for a number of selections of target convergence rates $\alpha \in[1,4]$. 


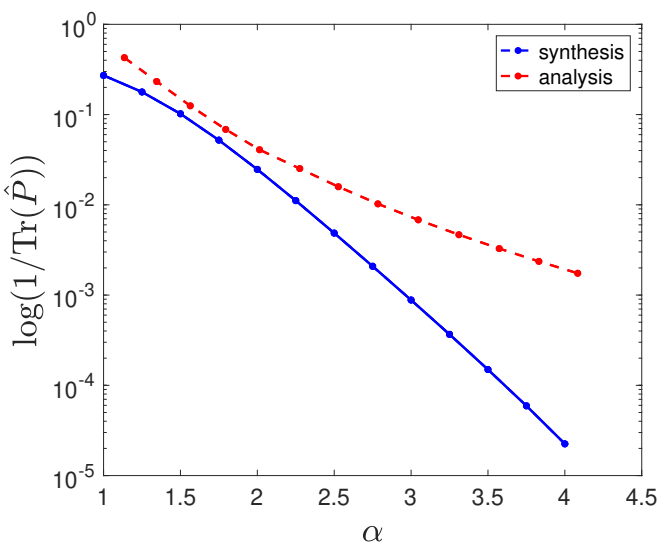

Fig. 5. Example 4: trade-off between the local convergence rate $\alpha$ and the optimization criterion $\operatorname{Tr}(\hat{P})$ in the synthesis conditions of Theorem 4 (blue curve) and the analysis conditions of Theorem 3.

The corresponding optimal costs $(\operatorname{Tr}(\hat{P}))^{-1}$ are represented in logarithmic scale by the blue dots in Figure 5. The tradeoff blue curve interpolates the evaluated points. We recall that the minimized trace of $\hat{P}$ is only an indication of the size of the basin of attraction $\mathcal{B}$, due to the property $\mathcal{E}(\hat{P}, 1) \subset$ $S(W, 1) \subset \mathcal{B}$.

To illustrate the reduced conservativeness of the analysis conditions of Theorem 3 and Proposition 3, as discussed in Remarks 3 and 6 , the red dots in the same figure report the actual local convergence rate $\alpha$ (easily evaluated as the maximum real part of the eigenvalues of $A+B K_{1}$ ) and the performance criterion $\operatorname{Tr}(\hat{P})$ evaluated by solving the optimization in (44) with the corresponding gains. As one may see, both the estimated $\alpha$ (by inequality (37) of Proposition 4) and the estimated trace of $\hat{P}$ (by inequality (50) of Proposition 5) in the synthesis conditions are significantly worse than those of the analysis case.

To illustrate the numerical conditioning of the obtained matrices, we report below the values of the gains obtained for $\alpha=3.5$, corresponding to

$$
\begin{aligned}
K_{1} & =\left[\begin{array}{cccc}
-0.0716 & 0.1813 & -2.2395 & -0.8175 \\
3.0745 & -1.2914 & 10.8585 & 12.8640
\end{array}\right] \\
K_{2} & =\left[\begin{array}{ll}
0.8391 & 0.0293 \\
0.7123 & 0.8391
\end{array}\right]
\end{aligned}
$$

inducing the following eigenvalues of $A+B K_{1} \in \mathbb{R}^{4 \times 4}$ : $-3.5733 \pm 0.5560 i ;-3.7880 \pm 1.6975 i$, which confirm the desired convergence rate higher than 3.5.

\section{CONCLUSIONS}

We proposed a new class of sign-indefinite quadratic forms for the stability analysis and control design of nonlinear control laws for linear systems with input saturation. Both local and global stability analysis and synthesis results are presented, in terms of convex optimization problems, using the arising piecewise smooth Lyapunov functions.

Numerical examples testify the substantial improvement over the existing results, showing that non-quadratic Lyapunov function have the ability to show global stability of some systems for which a quadratic function fails to exist. Moreover the numerical results highlight that improvements also emerge from relaxing the positivity of the quadratic form. For regional stability, a non-linear generalized sector condition is introduced and numerical examples illustrate the improvement of the estimates of the basin of attraction. Indeed, more general shapes than mere ellipsoids are obtained thanks to the implicit definition of the Lyapunov function.

Future work includes the investigation of efficient schemes for the solution of implicit functions induced by the use of nonlinear control laws. We will also investigate the design of dynamic non-linear output feedback laws and their application to the design of anti-windup compensators. Furthermore, another interesting direction is to consider other nonlinearities, possibly combined with saturation, as for example quantizers, backlash or friction functions, with the aim of improving the characterization of an asymptotically stable attractor.

\section{REFERENCES}

[1] Anonymous. Why the grippen crashed. Aerospace America, page 11 1994.

[2] M. Camlibel, J. Pang, and J. Shen. Lyapunov stability of complementarity and extended systems. SIAM Journal on Optimization, 17(4):10561101, 2007.

[3] F.H. Clarke. Optimization and Nonsmooth Analysis. Society for Industrial and Applied Mathematics, 1990.

[4] D. Dai, T. Hu, A.R. Teel, and L. Zaccarian. Piecewise-quadratic Lyapunov functions for systems with deadzones or saturations. Systems \& Control Letters, 58:365-371, 2009.

[5] M. C. de Oliveira and R. E. Skelton. Stability tests for constrained linear systems. In Perspectives in robust control, pages 241-257. Springer, 2001.

[6] R. Drummond, G. Valmorbida, and S. R. Duncan. Generalized absolute stability using Lyapunov functions with relaxed positivity conditions. IEEE Control Systems Letters, 2(2):207-212, April 2018.

[7] A.T. Fuller. In the large stability of relay and saturated control systems with actuator saturation. International Journal of Control, 10(4):457480, 1969.

[8] R. Goebel, R.G. Sanfelice, and A.R. Teel. Hybrid Dynamical Systems: modeling, stability, and robustness. Princeton University Press, 2012.

[9] J.M. Gomes da Silva Jr. and S. Tarbouriech. Antiwindup design with guaranteed regions of stability: an LMI-based approach. IEEE Transactions on Automatic Control, 50:106-111, 2005

[10] C. A. C. Gonzaga, M. Jungers, and J. Daafouz. Stability analysis of discrete-time Lur'e systems. Automatica, 48(9):2277 - 2283, 2012.

[11] T. Hu and Z. Lin. Control systems with actuator saturation: analysis and design. Birkhauser, Boston, 2001.

[12] T. Hu, A.R. Teel, and L. Zaccarian. Stability and performance for saturated systems via quadratic and nonquadratic Lyapunov functions. IEEE Transactions on Automatic Control, 51(11):1770-1786, 2006.

[13] T. Hu, A.R. Teel, and L. Zaccarian. Anti-windup synthesis for linear control systems with input saturation: achieving regional, nonlinear performance. Automatica, 44(2):512-519, 2008.

[14] H.K. Khalil. Nonlinear Systems. Prentice-Hall, 3rd edition, 2002.

[15] Y. Li and Z. Lin. A generalized piecewise quadratic Lyapunov function approach to estimating the domain of attraction of a saturated system. In IFAC-PapersOnLine, volume 48, pages 120-125, St Petersburg (Russia), 2015.

[16] Y. Li, Z. Lin, and N. Li. Stability and performance analysis of saturated systems using an enhanced max quadratic Lyapunov function. In Proceedings of the IFAC World Congress, pages 12353-12358, Toulouse, France, 2017. Elsevier.

[17] K.-Z. Liu and D. Akasaka. A partial parameterization of nonlinear output feedback controllers for saturated linear systems. Automatica, 50(1):233-239, 2014.

[18] G. Pipeleers, B. Demeulenaere, J. Swevers, and L. Vandenberghe. Extended LMI characterizations for stability and performance of linear systems. Systems \& Control Letters, 58:510-518, 2009.

[19] J. A. Primbs and M. Giannelli. Kuhn-tucker-based stability conditions for systems with saturation. IEEE Transactions on Automatic Control, 46(10):1643-1647, Oct 2001. 
[20] W.E. Schmitendorf and B.R. Barmish. Null controllability of linear systems with constrained controls. SIAM J. Contr. Opt., 18(4):327-345, 1980.

[21] E.D. Sontag. An algebraic approach to bounded controllability of linear systems. International Journal of Control, 39(1):181-188, 1984.

[22] G. Stein. Respect the unstable. IEEE Control Systems Magazine, 23(4):12-25, August 2003

[23] S. Tarbouriech, G. Garcia, J.M. Gomes da Silva Jr., and I. Queinnec. Stability and stabilization of linear systems with saturating actuators. Springer-Verlag London Ltd., 2011.

[24] S. Tarbouriech, C. Prieur, and J.M. Gomes da Silva Jr. Stability analysis and stabilization of systems presenting nested saturations. IEEE Transactions on Automatic Control, 51(8):1364-1371, 2006.

[25] A.R. Teel and L. Praly. On assigning the derivative of a disturbance attenuation control Lyapunov function. Mathematics of Control, Signals, and Systems (MCSS), 13(2):95-124, 2000.

[26] G. Valmorbida, R. Drummond, and S. R. Duncan. Regional analysis of slope-restricted Lurie systems. IEEE Transactions on Automatic Control, 64(3):1201-1208, March 2019.

[27] G. Valmorbida, A. Garulli, and L. Zaccarian. Regional $\mathcal{L}_{2 m}$ gain analysis for linear saturating systems. Automatica, 76:164 - 168, 2017.

[28] F. Wu, K.M. Grigoriadis, and A. Packard. Anti-windup controller design using linear parameter-varying control methods. International Journal of Control, 73(12):1104-1114, 2000

[29] V. A. Yakubovich, G. A. Leonov, and A. K. Gelig. Stability of Stationary Sets in Control Systems with Discontinuous Nonlinearities, volume 14 of Satbility, Vibartions and Control of Systems, Series A. World Scientific, 2004.

[30] L. Zaccarian and A.R. Teel. A common framework for anti-windup, bumpless transfer and reliable designs. Automatica, 38(10):1735-1744, 2002.

[31] L. Zaccarian and A.R. Teel. Modern anti-windup synthesis. Princeton University Press, Princeton, 2011.

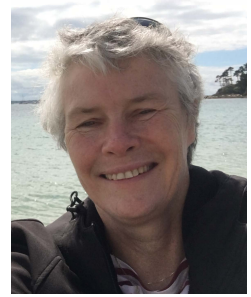

Isabelle Queinnec is currently CNRS researcher at LAAS-CNRS, Toulouse University. She received her $\mathrm{PhD}$ degree and HDR degree in automatic control in 1990 and 2000, respectively, from University Paul Sabatier, Toulouse. Her current research interests include constrained control and robust control of processes with limited information, with particular interest in applications on aeronautical systems, robotic, electronic, biochemical and environmental processes. She has been serving as member of the IFAC technical committees on "Biosystems and Bioprocesses" and on "Modelling and Control of Environmental Systems", respectively from 2002 and 2005 and of the IEEE CSS-CEB from 2013. She is currently AE for IEEE Transactions on Automatic Control. She is co-author of a book on saturated systems and of more than 50 journal papers, both in control theory and process engineering.

Sophie Tarbouriech received the Ph.D. and HDR degrees in automatic control from University Paul Sabatier, Toulouse, France, in 1991 and 1998, respectively. She is full-time researcher (Directeur de Recherche) in LAAS-CNRS, Toulouse. Her main research interests include analysis and control of linear and nonlinear systems with constraints (limited information), hybrid dynamical systems. She is currently Associate Editor for IEEE Transactions on Automatic Control, Automatica and European Journal of Control. She is also Senior Editor for IEEE Control Systems Letters and member of IFAC and IEEE Technical Committees

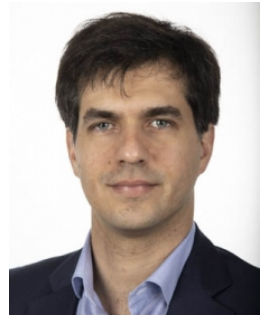

Giorgio Valmorbida received the $\mathrm{Ph} . \mathrm{D}$. degree in control theory from the Institut National des Sciences Appliquées, Toulouse, France, in 2010 for the research developed at the LAAS/CNRS. He has held post-doctoral research positions at the Università di Roma "Tor Vergata", Rome, Italy, from 2010 to 2012 and at the Department of Engineering Science, University of Oxford, Oxford, UK, from 2013 to 2015. He was a Junior Research Fellow at Somerville College, University of Oxford, Oxford, UK, from 2013 to 2015 . He is currently an Associate Professor in the L2S, CentraleSupélec, Gif-sur-Yvette, France. His main research interests include analysis and control of nonlinear systems with input constraints, hybrid systems, infinite-dimensional systems, and polynomial optimization.

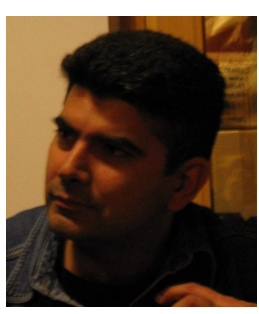

Luca Zaccarian (F'16) received the $\mathrm{Ph} . \mathrm{D}$. from the University of Roma Tor Vergata (Italy) in 2000 where he then became assistant and then associate professor. Since 2011 he is Directeur de Recherche at the LAAS-CNRS, Toulouse (France) and since 2013 he holds a part-time professor position at the University of Trento, Italy. Luca Zaccarian's main research interests include analysis and design of nonlinear and hybrid control systems, modeling and control of mechatronic systems. He was a recipient of the 2001 O. Hugo Schuck Best Paper Award given by the American Automatic Control Council. 